\title{
Asymmetric Autler-Townes-like doublets in laser-driven excitonic Fano resonance in biased superlattices: Role of many-body Coulomb exchange
}

\author{
Ken-ichi Hino, Ken Goto, and Nobuyuki Toshima \\ Institute of Materials Science, University of Tsukuba, 1-1-1 Tennodai, Tsukuba, Ibaraki 305-8573, Japan \\ (Received 20 March 2003; revised manuscript received 14 May 2003; published 28 January 2004)
}

\begin{abstract}
Transient four-wave mixing (FWM) spectra from excitonic Fano resonance (FR) states in biased superlattices are theoretically investigated by numerically solving the semiconductor Bloch equations adopting FR wave functions of the excitonic Wannier-Stark ladder as an expansion basis set. It is found that an AulterTownes-like doublet arises from resonant excitation of FR excitons, and shows conspicuous asymmetry in spectrally resolved FWM signals, the degree of which depends strongly upon a Fano- $q$ parameter. Furthermore, the many-body Coulomb exchange effects markedly modify the spectral profiles, so that either lobe of the doublet is considerably narrowed with accompanying reversal of the asymmetry pattern. The effects also shift the overall resonance spectra toward the high-energy side. The three findings of spectral narrowing, asymmetry reversal, and blue shift feature the present dressed FR system. The corresponding time-resolved spectra are also discussed from the viewpoint of the Rabi oscillation.
\end{abstract}

DOI: 10.1103/PhysRevB.69.035322

PACS number(s): 78.47.+p, 42.50.Md, 32.80.Dz

\section{INTRODUCTION}

Quantum interference of continuum states with embedded bound states, known as Fano resonance (FR), ${ }^{1}$ is one of the most fundamental phenomena occurring in diverse physical systems such as nuclei, atoms, molecules, and semiconductors. Interactions of laser fields with atomic FR, termed laserinduced autoionization (LIA), were intensively studied in the past, revealing several remarkable effects ${ }^{2}$ including the Fano zero and the confluence. ${ }^{3}$ Moreover, these interactions were also explored in the context of laser-induced continuum structure (LICS), in which atomic continuum states are structured by embedding a bound state into a previously unstructured continuum by the dressing interaction. ${ }^{4}$ As for semiconductor heterostructures, investigations in the direction of nonlinear responses from low-dimensional excitonic FR still remain scarce, ${ }^{5-9}$ though the corresponding linear spectra have been well understood both experimentally ${ }^{8,10,11}$ and theoretically. $^{12-17}$

On the other hand, a vestige of excitonic Rabi flopping induced by an intense pumping was successfully observed ${ }^{18}$ despite rapid polarization decay and possible excitationinduced dephasing (EID). ${ }^{19-23}$ Since this first observation, coherent nonlinear dynamics of resonantly excited excitons pertaining to pure bound states (not to FR) have been studied in both temporal and spectral domains, demonstrating characteristics of excitonic Rabi flopping, ${ }^{24-32}$ which is absent from atomic Rabi oscillation. ${ }^{33}$ Specifically, the excitonic Rabi energy is saliently enhanced due to internal renormalized fields arising from many-body Coulomb effects negligible in the atomic system. ${ }^{24-27}$ Moreover, it is likely that an exciton blue shift in energy attributed to repulsive excitonexciton interactions causes the higher-energy ac-Stark sideband to overlap with continuum states, resulting in modification of spectral intensities. ${ }^{28,29}$ In addition, a transition from the exciton-polariton doublet to an ac-Stark splitting and spectral modulation due to exciton continuum onset have been intensively studied in semiconductor microcavities. ${ }^{30-32}$
In this paper, we present transient four-wave mixing (FWM) spectra of the excitonic FR states induced by resonant pumping in biased superlattices [Wannier-Stark ladder (WSL)]. This subject, ${ }^{34}$ related to LIA as an atomic counterpart, is distinguished from the excitonic Rabi problem concerning the structureless continuum mentioned above. ${ }^{28,29}$ In the former, the FR wave function is usually complex because of its nature ascribable to a multichannel scattering problem, and sharply energy-dependent phase shifts associated with Fano couplings are inherently incorporated in the present continuum, rendering all associated FR transition matrices phase-sensitive complex numbers. On the other hand, in the latter, wave functions of the pure bound state and the structureless continuum are just real and do not provide any additional phase information for the related matrix elements. In this sense, the observed enhancement of the high-energy acStark sideband and the resulting asymmetric profile in the study of Ref. 29 would be considered due to overlap of energetically close bound and continuum states, rather than due to interference between the two.

It is thus speculated that the presence of the FR phase shifts largely modulates coherence of induced polarizations, and the modulation pattern of the spectra depends on a way of the Fano interference. As is seen later, the spectrally resolved FWM (SRFWM) signals in relatively strong excitation exhibit an Autler-Townes (AT)-like doublet ${ }^{33,35,36}$ with asymmetric amplitudes of the two sidebands. The asymmetry is ascribable to the modulation of polarization coherence due to the Fano couplings. The many-body Coulomb exchange is also strongly affected by these couplings through the relevant matrix elements with respect to the FR states. Furthermore, this effect possibly accelerates the spectral modification, leading to reversal of the asymmetry, narrowing of each sideband width, and blue shift.

Next a brief mention is made of the related studies to the present paper, other than the asymmetric ac-Stark doublet due to overlap between bound and continuum states remarked above. ${ }^{29}$ The first is on Fano-type interference in photoluminescence spectra of infrared-driven quantum 
wells, ${ }^{37}$ where a higher transition level of a two-level system is coupled to an additional metastable state, and the resulting emission spectra split into the AT doublet with equal amplitudes, namely, the symmetric doublet. This is akin to LICS in atomic physics rather than LIA in that this splitting is attributed to embedding an exciton bound state in continuum by laser irradiation. Moreover, it is governed by intraband dynamics in contrast with the present system ruled by interband dynamics. To the best of our knowledge, we are not aware of asymmetric doublet generation in this system. The second is on the asymmetric profile of the AT splitting arising from detuning in nonresonant pumping. Such phenomena are commonly known in atomic and molecular physics. ${ }^{33,38}$ Note that the asymmetry of a spectral shape focused in this article arises from resonant excitation. An excitation slightly detuned from the resonance would also result in asymmetry, however it has a combined origin from both effects of the detuning and the coherent modulation of an induced polarization due to FR.

In the present paper the semiconductor Bloch equations (SBE) are applied to the dressed FR problem concerned here for the excitonic WSL within the Hartree-Fock (HF) approximation, ${ }^{39}$ by expanding a microscopic polarization and a carrier population density in terms of an excitonic FR basis set. However, it is pointed out that the SBE's in the HF model tend to give incorrect results in that these underestimate the excitonic effects on the intraband dynamics, ${ }^{40}$ and overestimate contributions from continuum due to partial lack of excitonic correlation. ${ }^{29,32}$ Moreover, theories beyond the HF approximation have been presented, for instance, in the method of dynamic controlled truncation of the hierarchy of density matrices ${ }^{40}$ and the quasibosonic exciton model, ${ }^{41}$ indicating significance of the exciton-exciton correlation in intraband dynamics such as terahertz radiation. ${ }^{42-44}$ In addition, Coulomb memory signature is explored, ${ }^{45}$ and evidences for exciton-exciton correlations are found in heavyhole and light-hole oscillations. ${ }^{46}$ On the other hand, according to a recent theoretical work of Ciuti et al. ${ }^{47}$ incoherent scattering relevant to EID is drastically reduced in the regime of strong and resonant pumping of excitons, leading to little alteration from the HF dynamics at the more sophisticated dressed second-Born level.

Discussion of such Coulomb correlation effects is beyond the scope of this paper for the present. To the best of our knowledge, investigations toward the many-body Coulomb effect (including the HF effect) on nonlinear dynamics of FR excitons have still remained unexplored but a few experimental works ${ }^{6,7}$ suggesting its significance. Therefore it is considered worth showing the importance of the Coulomb collisions in the dressed FR system concerned here even within the mean-field approximation.

However, the authors should comment on the possible consequences of the deficiency in this approximation. The occurrence of the above-mentioned asymmetric AT-like doublet that is the primary result of the present paper would remain unaltered no matter whether the exciton correlation beyond the HF approximation is taken into account, since this arises from the interference between the microscopic interband polarization coherence and the FR, not from the many-body effect. Even without this effect, in fact, the asymmetry manifests itself, as is evidently seen later (Sec. III). On the other hand, it would be the way of modulation of the asymmetrically split sidebands in this doublet that will be likely governed by the correlation effect. As is shown below (Sec. IV), the modulation obtained at the HF level leads to detailed changes of the SRFWM spectra such as a narrowing of the split peaks. The many-body effect beyond this approximation would further alter the modulation pattern of the interband polarization through the intraband quantities such as population densities, since these quantities are sensitive to the exciton correlation. To recapitulate, it would be speculated that the SRFWM spectra of FR excitons are still globally featured by the manifestation of asymmetry even in a more sophisticated level of approximation including the correlation, whereas in contrast to it the detailed characters of the spectra are more or less modified by the correlation. We are now working on the study toward the sophisticated level of theory.

This paper is organized as follows. In Sec. II, the theoretical framework for numerical calculations is described. Prior to full numerical calculations based on SBE, in Sec. III, a simplified model without the many-body effect, which can be solved analytically, is presented for a qualitative analysis of the asymmetric AT-like doublet manifested in SRFWM signals. The associated time-resolved FWM (TRFWM) signals are also included. In Sec. IV, discussion is made on the results of the full numerical calculations including the manybody effect. Section V is a concluding remark. Moreover, three appendixes are included for supplementing the text. The atomic units are used throughout unless otherwise stated.

\section{THEORETICAL FRAMEWORK}

This section consists of linear FR spectra of WSL along with the associated wave function, and the SBE and the resulting mathematical expressions for FWM. The FR wave functions are employed as an expansion basis set of the SBE, as stated above. Therefore we begin with the FR spectra and discuss characteristics of the FR wave function from the point of view of the multichannel scattering problem. Next such a FR basis set is incorporated to the SBE formalism, and expressions of macroscopic polarizations for SRFWM and TRFWM are derived.

\section{A. Linear FR spectra}

There are no pure bound states in excitonic WSL, and all of the exciton states pertain to FR. ${ }^{8,12,13}$ The FR should be dealt with as the multichannel scattering (MCS) problem with imposition of proper scattering boundary conditions, ${ }^{14,15}$ differing from the conventional method. ${ }^{16,17}$ First of all, both the advantages of the MCS theory adopted in this paper and the drawbacks included in the conventional FR theories are summarized based on the preceding papers. ${ }^{14,15}$

In most theoretical studies ${ }^{16,17}$ resorting to the variational calculations, the obtained FR spectra were approximated simply by a set of pseudocontinuum states having discrete eigenvalues and being independent of a given FR energy. As 
the matter of course, the correct boundary condition was disregarded from the beginning, otherwise superseded implicitly by a vanishing boundary condition similarly to a boundstate problem. Furthermore, these studies were fairly crude due to necessity of incorporating an artificial phenomenological broadening parameter of a FR width for coping with the difficulty. Such approximations hamper detailed analysis of FR. For instance, it is impossible to extract phase information of FR and the related scattering matrix that will play significant roles in determining the shape of SRFWM signals in the present work (see Secs. II B, III, and IV). In these senses, these conventional works are incomplete.

On the other hand, the present method can overcome almost all difficulties annoying the conventional methods owing to the sophisticated numerical approach explored, namely, the $R$-matrix propagation technique combined with the adiabatic expansion, to be shown right below. The MCS calculation does not require any empirical broadening parameter, and hence it can provide the accurate natural FR widths and scattering wave functions with numerical stability. The only one drawback of the present FR theory is that a large size of data of the MCS wave functions composed of a lot of scattering channels has to be accumulated in a computer memory for its application to the nonlinear FR-exciton dynamics as an expansion basis set. To make feasible the calculation of Coulomb matrices needed in the dynamics, a couple of further approximations are introduced, as is shown in Sec. II B.

Now we move to the setup of FR wave functions of the WSL exciton. Let us begin with an excitonic Hamiltonian ascribable to a heavy-hole exciton without valence-band mixing and contributions from a light-hole exciton. Neglect of the valence-band mixing is well justified in the present system composed of relatively narrow quantum wells, namely, superlattices of $34 \AA-\mathrm{GaAs} / 17 \AA-\mathrm{Al}_{0.3} \mathrm{Ga}_{0.7} \mathrm{As}$. The light-hole exciton provides merely a minor effect on the FR spectra with slightly modifying higher-energy line shapes. ${ }^{8}$ Moreover, just the energetically lowest pair of electron $e$ and heavy-hole $h$ subbands are incorporated (the two-subband model), ignoring the Zener tunneling for simplicity. The excitonic Hamiltonian $H_{e x}$ is thus given by

$$
H_{e x}=-\frac{\nabla_{\boldsymbol{\rho}}^{2}}{2 m_{\|}}-V\left(\rho, z_{e}, z_{h}\right)+h_{w s l}\left(z_{e}, z_{h}\right),
$$

where an in-plane exciton radius vector is represented as $\boldsymbol{\rho}$, positions of $e$ and $h$ in the crystal-growth direction (the $z$ axis) are denoted as $z_{e}$ and $z_{h}$, respectively, and $m_{\|}$means an in-plane reduced mass of the exciton: $1 / m_{\|}=1 / m_{e} \|+1 / m_{h \|}$ with $m_{e(h) \|}$ an in-plane electron (hole) mass. A centerof-mass (c.m.) motion in the layer plane is neglected. $V$ is a Coulomb potential between electrons staying in conduction and valence bands, given by $V\left(\rho, z_{e}, z_{h}\right)$ $=1 /\left[\epsilon \sqrt{\rho^{2}+\left(z_{e}-z_{h}\right)^{2}}\right]$, with $\epsilon$ a static dielectric constant of a medium concerned. $h_{w s l}$ means a combined WSL Hamiltonian describing motions of $e$ and $h$ in the axial direction, including periodic potentials $u\left(z_{e}, z_{h}\right)$ of superlattices of both carriers and an interaction of these carriers with a static electric field $F_{0}$ applied in the growth direction: (a) Schematic diagram of the present WSL
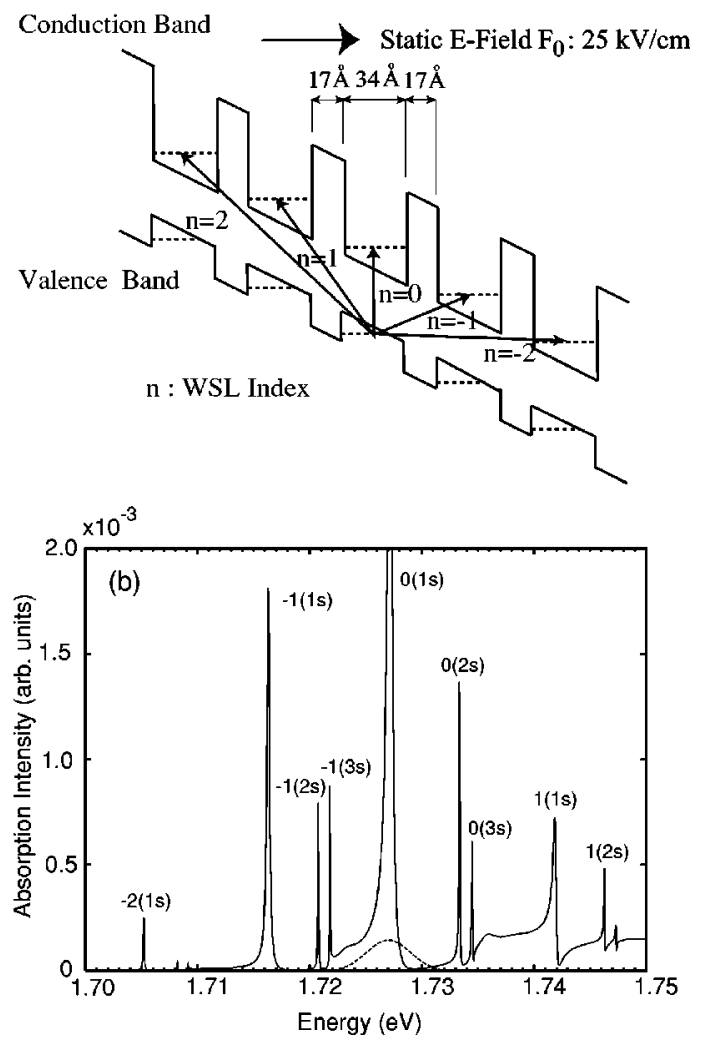

FIG. 1. (a) A schematic diagram of the present sample of WSL. (b) Linear absorption spectra of the sample under consideration vs photon energy. Peaks are denoted by $n(k s)$ with $n$ the WSL index and $k$ an exciton quantum number. The dashed curve stands for a frequency distribution of the pumping pulse with $\sigma_{2}=0.5 \mathrm{ps}$ and tuned to $\varepsilon_{r}=1.7273 \mathrm{eV}$.

$$
h_{w s l}=-\frac{1}{2 m_{e \perp}} \frac{\partial^{2}}{\partial z_{e}^{2}}-\frac{1}{2 m_{h \perp}} \frac{\partial^{2}}{\partial z_{h}^{2}}+u\left(z_{e}, z_{h}\right)-F_{0}\left(z_{e}-z_{h}\right) \text {, }
$$

where $m_{e \perp}$ and $m_{h \perp}$ are masses of $e$ and $h$, respectively, in the $z$ direction, and $F_{0}$ is set equal to $25 \mathrm{kV} / \mathrm{cm}$ hereafter unless otherwise stated. A schematic picture of the present WSL associated with $h_{w s l}$ is depicted in Fig. 1(a).

An exciton envelope function $\Psi$ following the effectivemass model satisfies the Wannier equation:

$$
\left(H_{e x}-E\right) \Psi(|\rho|, \Omega)=0,
$$

where $E$ is a given energy of the FR exciton, and $\Omega$ has been defined as a lump of the coordinates, $\Omega=\left(\hat{\boldsymbol{\rho}}, z_{e}, z_{h}\right)$, for the sake of typographical simplicity. According to the previous formalism of Refs. 14 and 15, the $\alpha$ th solution of $\Psi_{\alpha}$ is expanded with respect to a set of adiabatic channel functions $\left\{\Phi_{\mu}\right\}$ in the following:

$$
\Psi_{\alpha}(|\rho|, \Omega)=\frac{1}{\sqrt{|\rho|}} \sum_{\mu} \Phi_{\mu}(|\rho| ; \Omega) F_{\mu \alpha}^{E}(|\rho|) .
$$

Here $\Phi_{\mu}$ is an eigenfunction of the Schrödinger equation: 


$$
\left(h_{w s l}-V\right) \Phi_{\mu}(|\rho| ; \Omega)=U_{\mu}(|\rho|) \Phi(|\rho| ; \Omega),
$$

with the in-plane radius of the exciton $|\rho|$ fixed as an adiabatic parameter. The eigenvalue of $U_{\mu}$ stands for an adiabatic potential, being identical to the $\mu$ th WSL subband energy associated with $h_{w s l}$ at the limit of $|\rho| \rightarrow \infty$. An open (closed) channel $\mu$ is defined as an adiabatic channel satisfying $E>U_{\mu}(\infty)\left[E<U_{\mu}(\infty)\right]$. The subscript $\alpha$ of $\Psi_{\alpha}$ in Eq. (4) means the $\alpha$ th open channel, since the number of linearindependent solutions of Eq. (3) is equal to the number of open channels at each $E$. A radial wave function of $F_{\mu \alpha}$ is provided by use of the $R$-matrix propagation technique, as will be shown below. Upon this wave function, incoming scattering-wave boundary condition given by

$$
\begin{gathered}
F_{\mu \alpha}^{E} \rightarrow \chi_{\mu}^{(+)} \delta_{\mu \alpha}-\chi_{\mu}^{(-)} S_{\mu \alpha}^{(-)}(E) \quad \text { for } E>U_{\mu}(\infty), \\
F_{\mu \alpha}^{E} \rightarrow 0 \text { for } E<U_{\mu}(\infty)
\end{gathered}
$$

is imposed at $|\rho| \rightarrow \infty$, where $S_{\mu \alpha}^{(-)}$represents a scattering matrix ( $S$ matrix) including all information of exciton FR, and $\chi_{\mu}^{( \pm)}$are asymptotic matching wave functions given by plane waves: $\chi_{\mu}^{( \pm)}(|\rho|)=\exp \left( \pm i k_{\mu}|\rho|\right) N_{\mu}$. Here a constant of $N_{\mu}$ is determined by the energy-normalization condition

$$
\sum_{\mu} \int d|\rho|\left(F_{\mu \alpha}^{E}\right) * F_{\mu \beta}^{E^{\prime}}=\delta_{\alpha \beta} \delta\left(E-E^{\prime}\right),
$$

and $k_{\mu}=\sqrt{2 m_{\|}\left[E-U_{\mu}(\infty)\right]}$.

First, Eq. (5) is solved by expanding $\Phi_{\mu}$ in the following:

$$
\Phi_{\mu}(|\rho| ; \Omega)=\sum_{n} \varphi_{n}(\Omega) c_{n \mu}(|\rho|),
$$

with respect to a set of wave functions $\left\{\varphi_{n}\right\}$ defined by

$$
\varphi_{n}(\Omega)=\frac{1}{\sqrt{2 \pi}} \exp (\operatorname{im} \phi) \phi_{n}\left(z_{e}, z_{h}\right)
$$

Here $\phi_{n}$ is a WSL subband wave function, satisfying an equation

$$
\left(h_{w s l}-\varepsilon_{n}\right) \phi_{n}\left(z_{e}, z_{h}\right)=0,
$$

and a prefactor of $(1 / \sqrt{2 \pi}) \exp (i m \phi)$ represents a wave function of an in-plane angular motion of the $e$ and $h$ pair having an angular momentum $m$, with $\phi$ an angular coordinate associated with $\hat{\rho}$. In fact, an excitonic photoabsorption is dominated by the $s$-radial symmetry, namely, $m=0$, and henceforth it is taken. In Eq. (8), a coefficient $\left\{c_{n \mu}\right\}$ is determined at each $|\rho|$. In Eq. (10), $\varepsilon_{n}$ is given by $\varepsilon_{n}=\varepsilon_{0}$ $+n \omega_{B}$, where $n$ is a WSL index and $\omega_{B}$ is a Bloch frequency given by $\omega_{B}=F_{0} d$ with $d$ a lattice period of the superlattices concerned. $\phi_{n}$ is expressed as a combined WSL subband wave function defined by ${ }^{48}$

$$
\phi_{n}\left(z_{e}, z_{h}\right)=\frac{1}{\sqrt{N_{\text {site }}}} \sum_{l} \exp \left(i K_{z} l d\right) \phi_{n+l}^{e}\left(z_{e}\right) \phi_{l}^{h}\left(z_{h}\right),
$$

with $N_{\text {site }}$ the number of WSL sites included in the calculations, since there is an additional translational symmetry owing to the c.m. motion of a pair of $e$ and $h$ in the $z$ direction, which conserves the associated c.m.-momentum $K_{z}$. Hereafter it is understood that $K_{z}$ is set to zero, because only $K_{z}$ $=0$ contributes to photoabsorption. ${ }^{48} \phi_{l}^{e(h)}(z)$ is a WSL wave function of $e(h)$ having a site index of $l$. It is readily seen that there are relations that $\phi_{l}^{e(h)}(z+j d)=\phi_{l-j}^{e(h)}(z)$ and $\varepsilon_{l}=\varepsilon_{l-j}+j \omega_{B}$.

The wave functions $\left\{\phi_{l}^{e(h)}(z)\right\}$ are calculated by employing an expansion with respect to a set of basis-spline ( $B$-spline) functions $\left\{B_{i k}(z)\right\}$, where $B_{i k}(z)$ is the $i$ th normalized $B$ spline of an order $k$ with a knot sequence $\left\{z_{i}\right\} .{ }^{49} \mathrm{In}$ practice, we have set $k=10$. This problem easily ends up with a standard algebraic equation. It is seen that this procedure provides some advantages over the usually adopted recipe resorting to the Kane representation of WSL in combination with the Kronig-Penny model that is limited to a rectangular periodic potential. ${ }^{50}$ The $B$-spline method is flexible to any type of potentials, regardless of whether the potential is smooth or discontinuous. In fact, for the present rectangular potential, the $B$-spline method provides a large number of eigenvalues with high accuracy at one stroke by introducing knot multiplicity ${ }^{49}$ to a position corresponding to a boundary between a well and a barrier, where the potential is discontinuous. Within minor changes, the same computer program can be readily applied to, for instance, a sinusoidal potential that models WSL of optical lattices of ultracold atoms realized by laser-cooling technology. ${ }^{51-55}$ Furthermore, it is straightforward to apply this recipe to more formidable multiband problems relevant to the Zener tunneling. Extension of this method to other systems is beyond the scope of the present paper and it will be discussed elsewhere.

Now we are at the stage of seeking the radial wave functions $F_{\mu \alpha}^{E}$ by virtue of the $R$-matrix propagation technique. In accordance with this method, the following relation is obtained: ${ }^{14,15}$

$$
\begin{aligned}
F_{\mu \alpha}^{E}(|\rho|)= & \sum_{\xi}\left[G_{\mu \xi}\left(|\rho|,\left|\rho_{n+1}\right|\right) F_{\xi \alpha}^{E(D)}\left(\left|\rho_{n+1}\right|\right)\right. \\
& \left.-G_{\mu \xi}\left(|\rho|,\left|\rho_{n}\right|\right) F_{\xi \alpha}^{E(D)}\left(\left|\rho_{n}\right|\right)\right]
\end{aligned}
$$

which is defined in the $n$th sector $\left|\rho_{n}\right|<|\rho|<\left|\rho_{n+1}\right|$. Here $F_{\nu \alpha}^{E(D)}(|\rho|)$ is pertinent to a derivative of $F_{\nu \alpha}^{E}(|\rho|)$ with respect to $|\rho| . G_{\mu \xi}\left(|\rho|,\left|\rho_{n}\right|\right)$ is the $R$-matrix Green function propagating from $\left|\rho_{n}\right|$ to $|\rho|$. For more detail, consult Refs. 14 and 15 . The $R$ matrix of $R_{\mu \nu}$ is defined by

$$
R_{\mu \nu}(|\rho|)=\frac{1}{|\rho|} \sum_{\alpha} F_{\mu \alpha}^{E}(|\rho|)\left[F^{E(D)}(|\rho|)\right]_{\alpha \nu}^{-1} .
$$

Putting Eq. (13) into Eq. (12) yields a relation between $R\left(\left|\rho_{n+1}\right|\right)$ and $R\left(\left|\rho_{n}\right|\right)$ in terms of the $R$ matrix Green functions 


$$
\begin{aligned}
R\left(\left|\rho_{n+1}\right|\right)= & \widetilde{G}\left(\left|\rho_{n+1}\right|,\left|\rho_{n+1}\right|\right)-\widetilde{G}\left(\left|\rho_{n+1}\right|,\left|\rho_{n}\right|\right) \\
& \times \frac{1}{R\left(\left|\rho_{n}\right|\right)-\widetilde{G}\left(\left|\rho_{n}\right|,\left|\rho_{n}\right|\right)} \widetilde{G}\left(\left|\rho_{n}\right|,\left|\rho_{n+1}\right|\right),
\end{aligned}
$$

where matrix notations of $R$ and $\widetilde{G}$ have been adopted for simplicity, and

$$
\widetilde{G}\left(\left|\rho_{n}\right|,\left|\rho_{n^{\prime}}\right|\right)=\left(\sqrt{\left|\rho_{n}\right|}\right)^{-1} G\left(\left|\rho_{n}\right|,\left|\rho_{n^{\prime}}\right|\right)\left(\sqrt{\left|\rho_{n^{\prime}}\right|}\right)^{-1} .
$$

According to Eq. (14), the $R$ matrix can be made to propagate forward from the $n$th sector to the adjacent $(n+1)$ th sector in succession, and finally the $S$ matrix is extracted by comparing the $R$ matrix given at an asymptotic distance with the boundary conditions of Eq. (6).

A dipole moment $\tilde{\mu}_{\alpha}(E)$ for an interband transition to a FR state in the $\alpha$ th open channel at a given $E$ is provided by

$$
\tilde{\mu}_{\alpha}(E)=\mu_{0} \mu_{\alpha}(E) .
$$

Here $\mu_{0}$ means a dipole moment of an interband transition of a bulk crystal GaAs, $\mu_{\alpha}(E)$ represents a contribution of an exciton envelope function to $\tilde{\mu}_{\alpha}(E)$ :

$$
\mu_{\alpha}(E)=\sum_{\mu}^{N} F_{\mu \alpha}^{E}(0) \int d z\left[\Phi_{\mu}(0 ; \Omega)\right]_{z \equiv z_{e}=z_{h}},
$$

where $N$ stands for the total number of channels included and actually it can be set equal to $N_{\text {site }}$. Photoabsorption spectra $I(E)$ concerned here is expressed as

$$
I(E)=\sum_{\alpha}^{N_{o}}\left|\tilde{\mu}_{\alpha}(E)\right|^{2}
$$

with $N_{0}$ the total number of open channels at $E$. Photoabsorption spectra of the WSL-FR states in the present system is shown in Fig. 1(b), where each peak is labeled by $n(k s)$ with $n$ the WSL index and $k$ a hydrogenic principle quantum number of the exciton.

Before closing this section behavior of the radial wave functions for the open and closed channels in the vicinity of a FR energy of the main peak $0(1 s)$ in Fig. 1(b) is examined. For practical convenience to the following section, the adiabatic radial wave function $F_{\mu \alpha}^{E}$ mentioned above is transformed into the corresponding diabatic radial wave function $\hat{F}_{n \alpha}^{E}$ as

$$
\hat{F}_{n \alpha}^{E}(|\rho|)=\sum_{\mu} c_{n \mu}(|\rho|) F_{\mu \alpha}^{E}(|\rho|),
$$

where $c_{n \mu}$ has appeared in Eq. (8). In view of this, the total excitonic envelope function is recast into

$$
\Psi_{\alpha}(|\rho|, \Omega)=\frac{1}{\sqrt{|\rho|}} \sum_{n} \varphi_{n}(\Omega) \hat{F}_{n \alpha}^{E}(|\rho|)
$$

in place of Eq. (4). Since $\Phi_{\mu}(|\rho| ; \Omega)$ of Eq. (5) becomes identical to $\varphi_{n}(\Omega)$ at $|\rho| \rightarrow \infty$, the WSL index $n$ uniquely
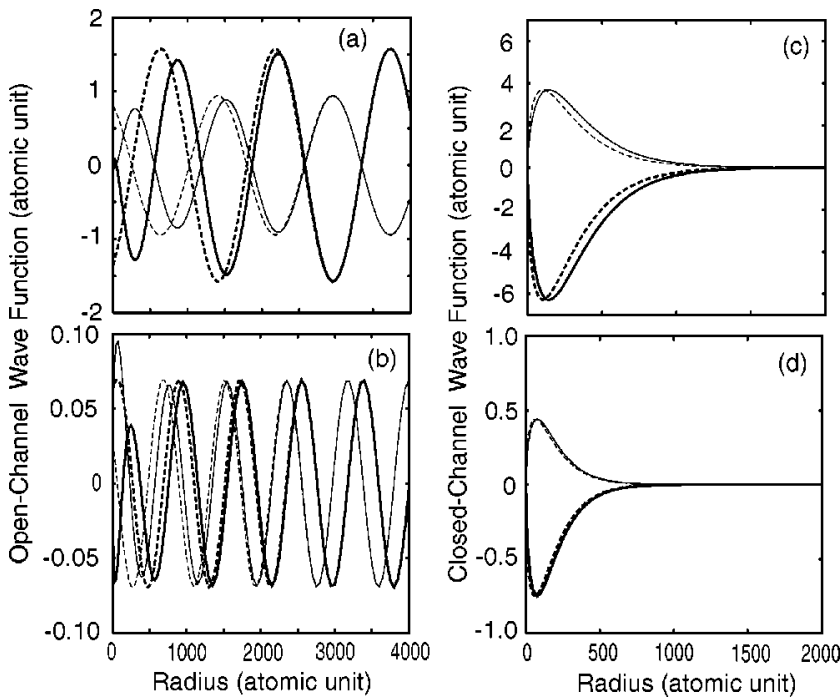

FIG. 2. Open-channel and closed-channel wave functions $\hat{F}_{n \alpha}^{E}(|\rho|)$ of Eq. (18) for the channel $\alpha=0$ and $E=1.7270 \mathrm{eV}$ close to the FR peak position as a function of an exciton radius $|\rho|$. (a) Wave functions for the highest-open channel of $n=\alpha$; (b) wave functions for the second highest-open channel of $n=\alpha-1$; (c) wave functions for the lowest-closed channel of $n=\alpha+1$; (d) wave functions for the second lowest-closed channel of $n=\alpha+2$. A thick (thin) line represents a real (imaginary) part of $\hat{F}_{n \alpha}^{E}(|\rho|)$. A solid line shows an accurate wave function obtained by numerical calculations, while a dashed line stands for an approximated wave function of Eqs. (20) and (21).

corresponds to the channel number $\mu$, and hence $n$ will be also termed as channel. Some of $\hat{F}_{n \alpha}^{E}(|\rho|)$ 's with $\alpha=0$ (a parent-band WSL) at $E=1.7270 \mathrm{eV}$ are depicted for open channels of $n=\alpha, \alpha-1$ in Figs. 2(a) and 2(b), respectively, and for closed channels of $n=\alpha+1, \alpha+2$ in Figs. 2(c) and 2(d), respectively. Solid lines represent the accurate wave function obtained by the numerical calculations presented above, where thick (thin) lines correspond to real (imaginary) parts. In $F_{0}=25 \mathrm{kV} / \mathrm{cm}$ of the present setup parameter, the highest open channel $(n=\alpha)$ is dominant over the lower one $(n=\alpha-1)$ by a factor of 30. Similarly, a contribution from the lowest closed channel $(n=\alpha+1)$ is greater than that of the higher one $(n=\alpha+2)$ by a factor of 10 . Therefore it suffices to incorporate just the single open channel of $n$ $=\alpha$ and the single closed channel of $n=\alpha+1$ into the SBE derived right below for evaluating FWM signals. Furthermore, dashed lines of Figs. 2(a) and 2(b) represent $\hat{F}_{n \alpha}^{E}(|\rho|)$ for $n$ that is open. This function is well approximated by

$$
\hat{F}_{n \alpha}^{E}(|\rho|) \approx \chi_{n}^{(+)}(|\rho|) \delta_{n \alpha}-\chi_{n}^{(-)}(|\rho|) S_{n \alpha}^{(-)}(E),
$$

apart that this breaks down in the vicinity of the origin. However, such an inaccuracy just causes a slight flaw in an integration over the whole space of $|\rho|$. Dashed lines of Figs. 2(c) and 2(d) also show $\hat{F}_{n \alpha}^{E}(|\rho|)$ for $n$ that is closed. This function is well approximated by

$$
\hat{F}_{n \alpha}^{E}(|\rho|) \approx \sqrt{|\rho|} \exp \left(-\kappa_{n}|\rho|\right) A_{n \alpha}^{(-)}(E),
$$

with $\kappa_{n}=\sqrt{2 m_{\|}\left|E-U_{n}(\infty)\right|}$. In Eqs. (20) and (21), $S_{n \alpha}^{(-)}(E)$ and $A_{n \alpha}^{(-)}(E)$ have been determined by the matching proce- 
dure in the numerical calculations. The use of these approximate expressions enables one to reduce entailing computational burdens considerably, when evaluating matrix elements associated with the many-body Coulomb exchange to be mentioned in the following section and Appendix A.

\section{B. SBE and FWM spectra}

The Hamiltonian of the system concerned here reads

$$
\begin{aligned}
H= & \sum_{l, \mathbf{k}, i=(c, v)} \varepsilon_{l \mathbf{k}}^{(i)} a_{l \mathbf{k}}^{(i) \dagger} a_{l \mathbf{k}}^{(i)}+\frac{1}{2} \\
& \times \sum_{l_{1}, l_{2}, l_{3}, l_{4}, \mathbf{k}, \mathbf{k}^{\prime}, \mathbf{q}(\neq \mathbf{q}), i, j=(c, v)} V_{l_{1} l_{2} l_{3} l_{4}}(\mathbf{q}) \\
& \times a_{l_{1} \mathbf{k}+\mathbf{k}+\mathbf{q}^{(i) \dagger}}^{(j) \dagger l_{2} \mathbf{k}^{\prime}-\mathbf{q}^{\prime} a_{l_{3} \mathbf{k}^{\prime}}} a_{l_{4} \mathbf{k}}^{(i)} \\
& -\sum_{l, l^{\prime}, \mathbf{k}} F(t)\left\{a_{l \mathbf{k}}^{(c) \dagger} a_{l^{\prime} \mathbf{k}}^{(v)} d_{l l^{\prime}}+\text { H.c. }\right\} .
\end{aligned}
$$

Here $a_{l \mathbf{k}}^{(i) \dagger}\left(a_{l \mathbf{k}}^{(i)}\right)$ is a creation (an annihilation) operator of the carrier $i$ (either a conduction electron $c$ or a valence-band electron $v$ ) at a site $l$ with a crystal momentum $\mathbf{k}$ in the layer plane. These satisfy anticommutation relations

$$
\begin{gathered}
{\left[a_{l \mathbf{k}}^{(i)}, a_{l^{\prime} \mathbf{k}^{\prime}}^{(j) \dagger}\right]_{+}=\delta_{i j} \delta_{l l^{\prime}} \delta\left(\mathbf{k}-\mathbf{k}^{\prime}\right),} \\
{\left[a_{l \mathbf{k}}^{(i)}, a_{l^{\prime} \mathbf{k}^{\prime}}^{(j)}\right]_{+}=\left[a_{l \mathbf{k}}^{(i) \dagger}, a_{l^{\prime} \mathbf{k}^{\prime}}^{(j) \dagger}\right]_{+}=0 .}
\end{gathered}
$$

The first term of Eq. (22) represents a sum of an energy of a WSL subband and a kinetic energy of the in-plane motion. The second term stands for electron-electron interactions accompanied by momentum transfer $\mathbf{q}$. The third term means a dipole interaction of the carrier with an external electric field of $F(t)$ with a dipole moment $d_{l l^{\prime}}$, where $\mathbf{k}$ dependence on $d_{l l}$ is neglected as usual, and H.c. is meant by taking a Hermitian conjugate of the first term in the curly brackets.

A density matrix is defined by

$$
\rho_{l l^{\prime} \mathbf{k}}^{(i j)}=\left\langle a_{l \mathbf{k}}^{(i) \dagger} a_{l^{\prime} \mathbf{k}}^{(j)}\right\rangle,
$$

where $\langle\cdots\rangle$ has been meant by taking an expectation value. Following a convention, it is convenient to rewrite the density matrix in the electron-hole representation by introducing operators $a_{l \mathbf{k}} \equiv a_{l \mathbf{k}}^{(c)}$ for the electron and $b_{l-\mathbf{k}} \equiv a_{l \mathbf{k}}^{(v) \dagger}$ for the hole. Thus the density matrices are recast into

$$
\begin{gathered}
\rho_{l l^{\prime} \mathbf{k}}^{(v c)}=\left\langle b_{l-\mathbf{k}} a_{l^{\prime} \mathbf{k}}\right\rangle \equiv P_{l l^{\prime} \mathbf{k}}, \\
\rho_{l l^{\prime} \mathbf{k}}^{(c c)}=\left\langle a_{l \mathbf{k}}^{\dagger} a_{l^{\prime} \mathbf{k}}\right\rangle \equiv N_{l l^{\prime} \mathbf{k}}^{(e)}, \\
\rho_{l l^{\prime} \mathbf{k}}^{(v v)}=\left\langle b_{l-\mathbf{k}} b_{l^{\prime}-\mathbf{k}}^{\dagger}\right\rangle \equiv \delta_{l l^{\prime}}-N_{l l^{\prime}-\mathbf{k}}^{(h)},
\end{gathered}
$$

where Eq. (23) has been employed in the last equation and $N_{l l^{\prime}-\mathbf{k}}^{(h)}=\left\langle b_{l^{\prime}-\mathbf{k}}^{\dagger} b_{l-\mathbf{k}}\right\rangle . P_{l l^{\prime} \mathbf{k}}$ indicates a microscopic polarization of an interband transition, a diagonal element of $N_{l l \mathbf{k}}^{(e / h)}$ stands for a population density of $e / h$, and an off-diagonal element $N_{l l^{\prime} \mathbf{k}}^{(e / h)}$ for $l \neq l^{\prime}$ represents a microscopic polarization of an intraband transition between WSL sites $l$ and $l^{\prime}$ of $e / h$.

The Heisenberg equation for the operators associated with $P_{l l^{\prime} \mathbf{k}}$ and $N_{l l^{\prime} \mathbf{k}}^{(e / h)}$ yields the following set of SBE within the random-phase approximation ${ }^{39}$

$$
\begin{aligned}
& i\left(\frac{d}{d t}+\frac{1}{T_{2}}\right) P_{l_{h} l_{e} \mathbf{k}}=\sum_{n}\left[\epsilon_{n l_{e} \mathbf{k}}^{(e)} P_{l_{h} n \mathbf{k}}+\epsilon_{l_{h} n \mathbf{k}}^{(h)} P_{n l_{e} \mathbf{k}}+\Omega_{n l_{h} \mathbf{k}} N_{n l_{e}}^{(e)}\right. \\
& \left.+\Omega_{l_{e^{n} \mathbf{k}}} N_{l_{h^{n}-\mathbf{k}}^{(h)}}^{(h)}-F(t) d_{l_{e} n} \delta_{l_{h^{n}}}\right] \\
& i\left(\frac{d}{d t}+\frac{1}{T_{1}^{(e)}}\right) \Delta N_{l_{e} l_{e}^{\prime} \mathbf{k}}^{(e)}=\sum_{n}\left[\epsilon_{n l_{e}^{\prime} \mathbf{k}}^{(e)} N_{l_{e} n \mathbf{k}}^{(e)}-\epsilon_{l_{e} n \mathbf{k}}^{(e)} N_{n l_{e}^{\prime} \mathbf{k}}^{(e)}\right. \\
& \left.+\Omega_{n l_{e} \mathbf{k}}^{\dagger} P_{n l_{e}^{\prime} \mathbf{k}}-\Omega_{l_{e}^{\prime} n \mathbf{k}} P_{l_{e} n \mathbf{k}}^{\dagger}\right], \\
& i\left(\frac{d}{d t}+\frac{1}{T_{1}^{(h)}}\right) \Delta N_{l_{h} l_{h}^{\prime}-\mathbf{k}}^{(h)}=\sum_{n}\left[\epsilon_{l_{h} n \mathbf{k}}^{(h)} N_{n l_{h}^{\prime}-\mathbf{k}}^{(h)}-\epsilon_{n l_{h}^{\prime} \mathbf{k}}^{(h)} N_{l_{h}^{n-\mathbf{k}}}^{(h)}\right. \\
& \left.+\Omega_{l_{h}^{\prime} n \mathbf{k}}^{\dagger} P_{l_{h} n \mathbf{k}}-\Omega_{n l_{h} \mathbf{k}} P_{n l_{h}^{\prime} \mathbf{k}}^{\dagger}\right] \text {. }
\end{aligned}
$$

Here an effective exciton energy $\epsilon_{l l^{\prime} \mathbf{k}}^{(e / h)}$ and an effective Rabi energy $\Omega_{l l^{\prime} \mathbf{k}}$ have been defined such that

$$
\begin{gathered}
\epsilon_{l_{e} l_{e}^{\prime} \mathbf{k}}^{(e)}=\left(\frac{\mathbf{k}^{2}}{2 m_{e} \|}+\varepsilon_{l_{e}}^{(e)}+E_{g}\right) \delta_{l_{e} l_{e}^{\prime}}+\Sigma_{l_{e} l_{e}^{\prime} \mathbf{k}}^{(e)}, \\
\epsilon_{l_{h} l_{h}^{\prime} \mathbf{k}}^{(h)}=\left(\frac{\mathbf{k}^{2}}{2 m_{h \|}}+\varepsilon_{l_{h}}^{(h)}\right) \delta_{l_{h} l_{h}^{l^{\prime}}}+\Sigma_{l_{h} l_{h}^{\prime} \mathbf{k}}^{(h)}+\Sigma_{l_{h} l_{h}^{\prime}}^{(c h)}, \\
\Omega_{l_{e} l_{h} \mathbf{k}}=F(t) d_{l_{e} l_{h}}+\Pi_{l_{e} l_{h} \mathbf{k}},
\end{gathered}
$$

with $\varepsilon_{l}^{[e(h)]}$ the WSL subband energy of $e(h), E_{g}$ a band gap of GaAs, and $\Sigma_{l_{h} l_{h}^{\prime}}^{(c h)}$ the Coulomb-hole self-energy. In Eqs. (27) and (28), $\Delta N_{l l^{\prime} \mathbf{k}}^{(e / h)}=N_{l l^{\prime} \mathbf{k}}^{(e / h)}-N_{l l^{\prime} \mathbf{k}}^{(e / h, e q)}$ with $N_{l l^{\prime} \mathbf{k}}^{(e /, e q)}$ a quasiequilibrium density. Moreover, the exchange selfenergy $\Sigma_{l l^{\prime} \mathbf{k}}^{(e / h)}$ and the internal renormalized field energy $\Pi_{l_{e} l_{h} \mathbf{k}}$ have been given by

$$
\begin{gathered}
\sum_{l_{e} l_{e}^{\prime} \mathbf{k}}^{(e)}=-\sum_{n_{e} n_{e}^{\prime} \mathbf{q}} V_{l_{e}^{\prime} l_{e} n_{e} n_{e}^{\prime}}(\mathbf{q}) N_{n_{e} n_{e}^{\prime} \mathbf{k}-\mathbf{q}}^{(e)}, \\
\sum_{l_{h} l_{h}^{\prime} \mathbf{k}}^{(h)}=-\sum_{n_{h^{n}}{ }^{\prime} \mathbf{q}} V_{l_{h}^{\prime} l_{h} n_{h} n_{h}^{\prime}}(\mathbf{q}) N_{n_{h^{n}} n_{h}^{\prime}-(\mathbf{k}-\mathbf{q})}^{(h)}, \\
\prod_{l_{e} l_{h} \mathbf{k}}=\sum_{n_{e} n_{h} \mathbf{q}} V_{l_{e} l_{h} n_{h} n_{e}}(\mathbf{q}) P_{n_{h} n_{e} \mathbf{k}-\mathbf{q} .} .
\end{gathered}
$$

$d_{l_{e} l_{h}}$ is a dipole moment of a transition between the $l_{e}$ th site of $e$ and the $l_{h}$ th site of $h$, given by $d_{l_{e} l_{h}}=\mu_{0} o_{l_{e} l_{h}}$, where $o_{l_{e} l_{h}}$ is the associated overlap matrix. In Eqs. (26)-(28), a phenomenological time of population relaxation $T_{1}^{(e / h)}$ and a dephasing time $T_{2}$ have been introduced. 
Since FR wave functions provided by Eqs. (19)-(21) have been prepared in the $\rho$ space, it is convenient to take the Fourier transformation of $P_{l l^{\prime} \mathbf{k}}$ and $N_{l l^{\prime} \mathbf{k}}^{(e / h)}$ from the $\mathbf{k}$ space into the $\rho$ space, that is,

$$
Z_{\mathbf{k}}=(2 \pi)^{-2} \int d \rho \exp (-i \mathbf{k} \cdot \rho) \widetilde{Z}_{\rho}
$$

for $Z_{\mathbf{k}}=P_{l l^{\prime} \mathbf{k}}$ or $N_{l l^{\prime} \mathbf{k}}^{(e / h)}$. Furthermore the transforms of $\widetilde{P}_{l l^{\prime} \rho}$ and $\widetilde{N}_{l l^{\prime} \rho}^{(e / h)}$ are expanded with respect to a complete set $\left\{\varphi_{l l^{\prime} \alpha}^{E}(\rho)\right\}$ as

$$
\begin{gathered}
\widetilde{P}_{l l^{\prime} \rho}(t)=\sum_{\alpha E} \varphi_{l l^{\prime} \alpha}^{E}(\rho) p_{\alpha E}(t), \\
\widetilde{N}_{l l^{\prime} \rho}^{(e / h)}(t)=\sum_{\alpha E} \varphi_{l l^{\prime} \alpha}^{E}(\rho) n_{\alpha E}^{(e / h)}(t) .
\end{gathered}
$$

Here $\varphi_{l l^{\prime} \alpha}^{E}$ is a projection of an excitonic three-dimensional envelope function $\Psi_{\alpha}^{E}$ of Eq. (19) onto the $\rho$ space, defined by

$$
\varphi_{l l^{\prime} \alpha}^{E}(\rho)=\left\langle\left\langle\phi_{l}^{(h)}\left(z_{h}\right) \phi_{l^{\prime}}^{(e)}\left(z_{e}\right) \mid \Psi_{\alpha}^{E}(|\rho|, \Omega)\right\rangle\right\rangle_{z_{e}, z_{h}},
$$

with $\langle\langle\cdots\rangle\rangle_{z_{e}, z_{h}}$ representing integrations over two variables of $z_{e}$ and $z_{h}$. The closure relation

$$
\sum_{\alpha E}\left|\varphi_{l l^{\prime} \alpha}^{E}(\rho)\right\rangle\left\langle\varphi_{l l^{\prime} \alpha}^{E}\left(\rho^{\prime}\right)\right|=\delta\left(\rho-\rho^{\prime}\right)
$$

is readily verified by use of the similar closure relation for $\left\{\Psi_{\alpha}^{E}\right\}$

$$
\sum_{\alpha E}\left|\Psi_{\alpha}^{E}(\mathbf{r})\right\rangle\left\langle\Psi_{\alpha}^{E}\left(\mathbf{r}^{\prime}\right)\right|=\delta\left(\mathbf{r}-\mathbf{r}^{\prime}\right)
$$

with $\mathbf{r}^{\left({ }^{\prime}\right)} \equiv\left(|\rho|^{\left({ }^{\prime}\right)}, \Omega^{\left({ }^{\prime}\right)}\right)$.

In passing, a remark is made on the exciton basis set of Eq. (39) used in the expansion of Eqs. (36) and (37). A merit of employing this basis set is that the Coulomb interaction between $e$ and $h$ within a single exciton is automatically included in theory, whereas a part of this effect is conventionally missing in the SBE with the HF model using the free-particle basis set for each of $e$ and $h$. In this spirit, many authors have been working with the exciton basis set. ${ }^{43,44,56-58}$ However, it should be noted that the present study is still done within the HF approximation and the higher-order exciton correlation is not incorporated, as is described in Sec. I.

Putting Eqs. (36) and (37) into the SBE of Eqs. (26)-(28) in view of Eq. (35) yields a set of SBE for unknown coefficients of $p_{\alpha E}$ and $n_{\alpha E}^{(e / h)}$ as follows:

$$
\begin{aligned}
i\left(\frac{d}{d t}+\frac{1}{T_{2}}\right) p_{\alpha E}(t)= & \sum_{\gamma E^{\prime \prime}} \mathcal{E}_{\alpha E ; \gamma E^{\prime \prime}}(t) p_{\gamma E^{\prime \prime}}(t) \\
& +\sum_{\gamma E^{\prime \prime}, s=e, h} \Omega_{\alpha E ; \gamma E^{\prime \prime}}(t) n_{\gamma E^{\prime \prime}}^{(s)}(t) \\
-(2 \pi)^{2} \Omega_{R}(t) \mu_{\alpha}^{*}(E), & \\
i\left(\frac{d n_{\alpha E}^{(e / h)}(t)}{d t}+\frac{\Delta n_{\alpha E}^{(e / h)}(t)}{\left.T_{1}^{(s)}\right)}=\right. & \sum_{\beta} u_{\alpha \beta E}^{(e / h)} n_{\beta E}^{(e / h)}(t) \\
& +\sum_{\gamma E^{\prime \prime}}\left[\Omega_{\alpha E ; \gamma E^{\prime \prime}}^{\prime}(t) p_{\gamma E^{\prime \prime}}(t)\right. \\
& \left.-\Omega_{\alpha E ; \gamma E^{\prime \prime}}^{\prime \prime}(t) p_{\gamma E^{\prime \prime}}^{*}(t)\right] .
\end{aligned}
$$

Here $\mathcal{E}_{\alpha E ; \gamma E^{\prime \prime}}$ being an effective exciton energy, and $\Omega_{\alpha E ; \gamma E^{\prime \prime}}, \Omega_{\alpha E ; \gamma E^{\prime \prime}}^{\prime}$, and $\Omega_{\alpha E ; \gamma E^{\prime \prime}}^{\prime \prime}$ being effective Rabi energies read

$$
\begin{aligned}
\mathcal{E}_{\alpha E ; \gamma E^{\prime \prime}}= & \left(E+\sum^{(c h)}\right) \delta_{\alpha \gamma} \delta\left(E-E^{\prime \prime}\right) \\
& -\sum_{\beta E^{\prime}, s=e, h} n_{\beta E^{\prime}}^{(s)} \mathcal{V}_{\alpha E ; \beta E^{\prime} ; \gamma E^{\prime \prime}},
\end{aligned}
$$

$$
\Omega_{\alpha E ; \gamma E^{\prime \prime}}=\Omega_{R} \delta_{\alpha \gamma} \delta\left(E-E^{\prime \prime}\right)+\sum_{\beta E^{\prime}} p_{\beta E^{\prime}} \mathcal{V}_{\alpha E ; \beta E^{\prime} ; \gamma E^{\prime \prime}}
$$

$$
\Omega_{\alpha E ; \gamma E^{\prime \prime}}^{\prime}=\Omega_{R} \delta_{\alpha \gamma} \delta\left(E-E^{\prime \prime}\right)+\sum_{\beta E^{\prime}} p_{\beta E^{\prime}} \mathcal{V}_{\alpha E ; \beta E^{\prime} ; \gamma E^{\prime \prime}}^{\prime},
$$

and

$$
\begin{aligned}
\Omega_{\alpha E ; \gamma E^{\prime \prime}}^{\prime \prime}= & \sum_{\beta E^{\prime}}\left[\Omega_{R}\left(-\frac{S_{\beta \alpha}^{(-) *}(E)+S_{\alpha \beta}^{(-)} *(E)}{2}\right)\right. \\
& \left.\times \delta_{\beta \gamma} \delta\left(E-E^{\prime}\right) \delta\left(E-E^{\prime \prime}\right)+p_{\beta E^{\prime}} \mathcal{V}_{\alpha E ; \beta E^{\prime} ; \gamma E^{\prime \prime}}^{\prime \prime}\right]
\end{aligned}
$$

respectively. $\mathcal{V}_{\alpha E ; \beta E^{\prime} ; \gamma E^{\prime \prime}}$ represents the HF Coulomb exchange integral given by

$$
\begin{aligned}
\mathcal{V}_{\alpha E ; \beta E^{\prime} ; \gamma E^{\prime \prime}}= & (2 \pi)^{-2} \sum_{j_{h} k_{e} n_{e} l_{e} l_{h}} \int d \mathbf{x} V_{n_{e} l_{h} j_{h} k_{e}}(\mathbf{x}) \varphi_{j_{h} k_{e} \beta}^{E^{\prime}}(\mathbf{x}) \\
& \times \int d \rho \varphi_{l_{h} l_{e} \alpha}^{E *}(\rho) \varphi_{n_{e} l_{e}^{\gamma}}^{E^{\prime \prime}}(\rho-\mathbf{x})
\end{aligned}
$$

Similarly, $\mathcal{V}_{\alpha E ; \beta E^{\prime} ; \gamma E^{\prime \prime}}^{\prime}$ is provided by

$$
\begin{aligned}
\mathcal{V}_{\alpha E ; \beta E^{\prime} ; \gamma E^{\prime \prime}}^{\prime}= & (2 \pi)^{-2} \sum_{j_{h} k_{e} n_{h} l_{e} l_{e}^{\prime}} \int d \mathbf{x} V_{n_{h} l_{e} k_{e} j_{h}}(\mathbf{x}) \varphi_{j_{h} k_{e} \beta}^{E^{\prime} *}(\mathbf{x}) \\
& \times \int d \rho \varphi_{l_{e} l_{e}^{l^{\prime} \alpha}}^{E^{*}}(\rho) \varphi_{n_{h} l_{e} \gamma}^{E^{\prime \prime}}(\rho-\mathbf{x})
\end{aligned}
$$


and $\mathcal{V}_{\alpha E ; \beta E^{\prime} ; \gamma E^{\prime \prime}}^{\prime \prime}$ is obtained by replacing $\varphi_{j_{h} k_{e} \beta}^{E^{\prime} *}(\mathbf{x})$ and $\varphi_{n_{h} l_{e} \gamma}^{E^{\prime \prime}}(\rho-\mathbf{x})$ in Eq. (48) by their complex conjugates. The Coulomb matrix element of $V_{l_{1} l_{2} l_{3} l_{4}}(\mathbf{x})$ is understood to be the Fourier transform of $V_{l_{1} l_{2} l_{3} l_{4}}(\mathbf{q})$ appearing in Eq. (22) despite using the same notations. The presence of the factor, $-\left[S_{\beta \alpha}^{(-) *}(E)+S_{\alpha \beta}^{(-) *}(E)\right] / 2$, of Eq. (46) attributes to an integral $\int d \mathbf{r} \Psi_{\alpha}^{E *}(\mathbf{r}) \Psi_{\beta}^{E^{\prime} *}(\mathbf{r}) . \quad \Delta n_{\alpha E}^{(e / h)}=n_{\alpha E}^{(e / h)}-n_{\alpha E}^{(e / h, e q)}$, which is associated with $\Delta N_{l l^{\prime} \mathbf{k}}^{(e / h)}$ mentioned above, and $\Sigma_{l_{h} l_{h}^{\prime}}^{(c h)}$ has been set equal to $\Sigma^{(c h)} \delta_{l_{h} l_{h}^{\prime}}$ for simplicity. $\mu_{\alpha}(E)$ has been expressed here by

$$
\mu_{\alpha}(E)=\frac{1}{\sqrt{N_{\text {site }}}} \sum_{\lambda \lambda^{\prime}}\left\langle\phi_{\lambda}^{(e)}(z) \mid \phi_{\lambda^{\prime}}^{(h)}(z)\right\rangle \varphi_{\lambda \lambda^{\prime} \alpha}^{E}(\rho=\mathbf{0}),
$$

which coincides with Eq. (16), and $\Omega_{R}=\mu_{0} F$ is the Rabi frequency. Note that $n_{\alpha E}^{(e / h)}$ is a complex quantity in this FR case, inasmuch as $\varphi_{l l^{\prime} \alpha}^{E}$ is always complex as seen in Fig. 2. In addition, $u_{\alpha \beta E}^{(e / h)}=\Sigma_{l l^{\prime}}\left(l-l^{\prime}\right) \omega_{B}\left\langle\varphi_{l l^{\prime} \alpha}^{E} \mid \varphi_{l l^{\prime} \beta}^{E}\right\rangle$. For simplicity of practical numerical calculations, it is assumed hereafter that $T_{1}^{(e)}=T_{1}^{(h)} \equiv T_{1}$ and $n_{\alpha E}^{(e, e q)}=n_{\alpha E}^{(h, e q)} \equiv n_{\alpha E}^{(e q)}$ so that $n_{\alpha E}^{(e)}$ $=n_{\alpha E}^{(h)} \equiv n_{\alpha E}$.

In SBE of Eqs. (26)-(28), the laser field $F(t)$ is given within the rotating-wave approximation by

$$
F(t)=\frac{1}{2}\left(\sum_{j=1,2} F_{j}(t) \exp \left(i\left[\mathbf{K}_{j} \cdot \mathbf{R}-\omega_{j} t\right]\right)\right),
$$

where $F_{j}$ is an envelope of the $j$ th pulse $(j=1$ for a probe beam and $j=2$ for a pump one) with $\mathbf{K}_{j}$ and $\omega_{j}$ a momentum and a center frequency of light, respectively, and $\mathbf{R}$ being position conjugate to $\mathbf{K}_{j}$. Hereafter each pulse is considered Gaussian, having a temporal width $\sigma_{j}$ and the maximum amplitude $F_{j 0}$. That is,

$$
F_{j}(t)=F_{j 0} g_{j}(t)
$$

where

$$
g_{1}(t)=\exp \left(-\frac{(t+\tau)^{2}}{\sigma_{1}^{2}}\right), \quad g_{2}(t)=\exp \left(-\frac{t^{2}}{\sigma_{2}^{2}}\right),
$$

with $\tau$ a delayed time.

Here brief mention is made of recipes of practical computations, above all, how to evaluate the HF Coulomb exchange integrals of $\mathcal{V}_{\alpha E ; \beta E^{\prime} ; \gamma E^{\prime \prime}}, \quad \mathcal{V}_{\alpha E ; \beta E^{\prime} ; \gamma E^{\prime \prime}}^{\prime}$ and $\mathcal{V}_{\alpha E ; \beta E^{\prime} ; \gamma E^{\prime \prime}}^{\prime \prime}$ Generally speaking, many channels have to be included in the calculation for each $E$ because of the multichannel nature of FR, in addition that continuum spectra over $E$ have to be taken into account. Nevertheless, as far as the present WSL-FR system is concerned, we can safely reduce the number of channels incorporated by bearing in mind the behavior of radial wave functions shown in Fig. 2 . The highest-lying open channel and the lowest-lying closed channel are dominant compared with other distant channels. Therefore it is considered that just this pair of channels at every $E$ suffice to obtain accurate results and others are neglected. This is termed a dominant-channel approximation from now on. Moreover, the Coulomb matrix element of $V_{l_{1} l_{2} l_{3} l_{4}}(\mathbf{x})$ appearing in Eqs. (47) and (48) is explicitly expressed as

$$
\begin{aligned}
V_{l_{1} l_{2} l_{3} l_{4}}(\mathbf{x})= & \left\langle\left\langle\phi_{l_{1}}^{(i)}(z) \phi_{l_{2}}^{(j)}\left(z^{\prime}\right)\left|V\left(\mathbf{x}, z, z^{\prime}\right)\right|\right.\right. \\
& \left.\left.\times \phi_{l_{3}}^{(j)}\left(z^{\prime}\right) \phi_{l_{4}}^{(i)}(z)\right\rangle\right\rangle_{z, z^{\prime}}
\end{aligned}
$$

with $i, j=e$ or $h$. At an asymptotic distance, $|\mathbf{x}|\rangle\rangle 1$, it becomes

$$
V_{l_{1} l_{2} l_{3} l_{4}}(\mathbf{x}) \rightarrow V(\mathbf{x}) \delta_{l_{1} l_{4}} \delta_{l_{2} l_{3}},
$$

where $V(\mathbf{x})=1 /(\epsilon|\mathbf{x}|)$. It is supposed that the matrix element concerned here is well approximated by its asymptotic form. This approximation would be accurate in the present WSL having a relatively strong $F_{0}$ owing to a localization nature of the WSL wave function. It is termed the asymptoticpotential approximation. Based on these two approximations, namely, the dominant-channel one and the asymptoticpotential one, in addition to employing Eqs. (20) and (21), the HF Coulomb exchange integral is made feasible. For more details of evaluation of the integral, consult Appendix A.

To evaluate FWM signals, first one takes spatial Fourier transformations of $p_{\alpha E}$ and $n_{\alpha E}$ as follows:

$$
p_{\alpha E}=\sum_{l} \exp (i[(\mathbf{K}+l \Delta \mathbf{K}) \cdot \mathbf{R}]) p_{\alpha E}^{(l)}
$$

and

$$
n_{\alpha E}=\sum_{l} \exp (i l \Delta \mathbf{K} \cdot \mathbf{R}) n_{\alpha E}^{(l)},
$$

respectively, where $\mathbf{K}=\left(\mathbf{K}_{1}+\mathbf{K}_{2}\right) / 2$ and $\Delta \mathbf{K}=\left(\mathbf{K}_{1}-\mathbf{K}_{2}\right) / 2$. Putting these expressions into the SBE of Eqs. (41) and (42) yields a set of coupled equations for $p_{\alpha E}^{(l)}$ and $n_{\alpha E}^{(l)}$, where the summation over $l$ is limited to $l_{\text {max }}$, i.e., $|l| \leqslant l_{\text {max }}$. A macroscopic polarization $P$ diffracted in the $2 \mathbf{K}_{2}-\mathbf{K}_{1}$ direction in the time domain is cast into the form

$$
P(t, \tau)=\mu_{0} \sum_{\alpha E} \mu_{\alpha}(E) p_{\alpha E}^{(-3)}(t) .
$$

The macroscopic polarization in the frequency domain is given by

$$
\widetilde{P}(\omega, \tau)=\mu_{0} \sum_{\alpha E} \mu_{\alpha}(E) \tilde{p}_{\alpha E}^{(-3)}(\omega),
$$

where $p_{\alpha E}^{(-3)}(t)=\int d \omega \exp (-i \omega t) \tilde{p}_{\alpha E}^{(-3)}(\omega)$. TRFWM and SRFWM signals are given by

$$
I^{T R F W M}(t, \tau)=|P(t, \tau)|^{2}, \quad I^{S R F W M}(\omega, \tau)=|\widetilde{P}(\omega, \tau)|^{2} .
$$




\section{ANALYTIC MODEL WITHOUT THE COULOMB EXCHANGE}

Prior to showing results of the fully numerical calculations based on Eqs. (41) and (42), just for qualitative understanding of the underlying physics, it is worth obtaining an analytic expression of $p_{\alpha E}^{(-3)}$ under some approximations to be made right below. Note that results obtained here are more or less modified in more quantitative discussion made in the following section. The many-body effects relevant to $\mathcal{V}_{\alpha E ; \beta E^{\prime} ; \gamma E^{\prime \prime}}$ are neglected in SBE. Moreover, the dominantchannel approximation discussed in Sec. II B is adopted. Thus the resulting SBE from Eqs. (41) and (42) is expressed as

$$
\begin{aligned}
i\left(\frac{d}{d t}+\frac{1}{T_{2}}\right) \bar{p}_{\alpha E}^{(l)}= & \varepsilon \bar{p}_{\alpha E}^{(l)}+\Omega_{1}(t)\left[2 \bar{n}_{\alpha E}^{(l-1)}-(2 \pi)^{2} \bar{\mu}_{\alpha}^{*}(E) \delta_{l 1}\right] \\
& +\Omega_{2}(t)\left[2 \bar{n}_{\alpha E}^{(l+1)}-(2 \pi)^{2} \bar{\mu}_{\alpha}^{*}(E) \delta_{l-1}\right]
\end{aligned}
$$

$$
\begin{aligned}
i\left(\frac{d}{d t}+\frac{1}{T_{1}}\right) \bar{n}_{\alpha E}^{(l)}= & \Omega_{1}^{*}(t) \bar{p}_{\alpha E}^{(l+1)}+\Omega_{2}^{*}(t) \bar{p}_{\alpha E}^{(l-1)} \\
& -\Omega_{1}(t) \bar{p}_{\alpha E}^{(1-l) *}-\Omega_{2}(t) \bar{p}_{\alpha E}^{(-l-1) *},
\end{aligned}
$$

where scaled notations have been introduced, defined as $\bar{p}_{\alpha E}^{(l)}=-i\left[S_{\alpha \alpha}^{(-)}\right]^{1 / 2} p_{\alpha E}^{(l)}, \bar{n}_{\alpha E}^{(l)}=-i\left[S_{\alpha \alpha}^{(-)}\right]^{1 / 2} n_{\alpha E}^{(l)}$ and $\bar{\mu}_{\alpha}(E)=$ $-i\left[S_{\alpha \alpha}^{(-)}\right]^{1 / 2} \mu_{\alpha}(E) \quad$ with $\quad \varepsilon=E+\Sigma^{(c h)}, \quad$ and $\Omega_{j}(t)$ $=\Omega_{j 0} g_{j}(t) \exp \left(-i \omega_{j} t\right)$ with $\Omega_{j 0}=\mu_{0} F_{j 0} / 2$ for $j=1,2$. For further simplicity, it is assumed that $l_{\max }=3, F_{10}\left\langle\left\langle F_{20}\right.\right.$, $\left.\left.\sigma_{2}\right\rangle\right) 1$, and $T_{1}=T_{2} \equiv 2 / \Gamma$, in which the first equation is valid as far as the second one holds, showing a weak probe limit, and the third one is justified in the narrow-band limit of the pumping laser. Contributions from $\bar{n}_{\alpha E}^{(0)}$ and $\bar{n}_{\alpha E}^{(2)}$ are omitted, since the fact that $\left.\left.\left|\bar{n}_{\alpha E}^{(-2)}\right|\right\rangle\right\rangle\left|\bar{n}_{\alpha E}^{(0)}\right|,\left|\bar{n}_{\alpha E}^{(2)}\right|$ has been confirmed by the full numerical calculations. A contribution from $\bar{p}_{\alpha E}^{(3)}$ is also neglected, which is justified for the probe weak enough. Under these approximations, the resulting equations, just including $\bar{p}_{\alpha E}^{( \pm 1)}, \bar{n}_{\alpha E}^{(-2)}$, and $\bar{p}_{\alpha E}^{(-3)}$, end up with

$$
\begin{gathered}
i\left(\frac{d}{d t}+\Gamma / 2\right) \bar{p}_{\alpha E}^{(1)}=\varepsilon \bar{p}_{\alpha E}^{(1)}-(2 \pi)^{2} \Omega_{1} \bar{\mu}_{\alpha}^{*}(E) \\
i\left(\frac{d}{d t}+\Gamma / 2\right) \bar{p}_{\alpha E}^{(-1)}=\varepsilon \bar{p}_{\alpha E}^{(-1)}-(2 \pi)^{2} \Omega_{2} \bar{\mu}_{\alpha}^{*}(E) \\
i\left(\frac{d}{d t}+\Gamma / 2\right) \bar{p}_{\alpha E}^{(-3)}=\varepsilon \bar{p}_{\alpha E}^{(-3)}+2 \Omega_{2} \bar{n}_{\alpha E}^{(-2)} \\
i\left(\frac{d}{d t}+\Gamma / 2\right) \bar{n}_{\alpha E}^{(-2)}=\Omega_{1}^{*} \bar{p}_{\alpha E}^{(-1)}+\Omega_{2}^{*} \bar{p}_{\alpha E}^{(-3)}-\Omega_{2} \bar{p}_{\alpha E}^{(1) *}
\end{gathered}
$$

The first and the second expressions indicate linear polarizations induced by the pulses 1 and 2 , respectively. They are readily solved, leading to

$$
\begin{aligned}
\bar{p}_{\alpha E}^{(j)}= & (2 \pi)^{2} \exp [-i(\varepsilon-i \Gamma / 2) t] \bar{\mu}_{\alpha}^{*}(E) \\
& \times \int d \eta \widetilde{\Omega}_{j}(\eta) \frac{\exp [i(\varepsilon-\eta-i \Gamma / 2) t]}{\varepsilon-\eta-i \Gamma / 2},
\end{aligned}
$$

where $j= \pm 1$ and

$$
\Omega_{j}(t)=\int d \eta \exp (-i \eta t) \widetilde{\Omega}_{j}(\eta),
$$

with $\Omega_{-1} \equiv \Omega_{2}$. The third and the fourth equations of Eq. (62) are coupled equations between $\bar{p}_{\alpha E}^{(-3)}$ and $\bar{n}_{\alpha E}^{(-2)}$ with inhomogeneous terms given by Eq. (63). Hence these can be reduced to a standard second-order differential equation of $\bar{p}_{\alpha E}^{(-3)}$, being of the form

$$
\frac{d^{2} y}{d t^{2}}+i\left(\omega_{2}-\varepsilon\right) \frac{d y}{d t}+2 \Omega_{20}^{2} y=X(t),
$$

where $y(t) \equiv \exp [i(\varepsilon-i \Gamma / 2) t] \bar{p}_{\alpha E}^{(-3)}(t)$ and the inhomogeneous term $X$ has been written as

$$
\begin{aligned}
X(t)= & -2 \Omega_{2}(t) \exp (i \varepsilon t)\left\{\Omega_{1}^{*}(t)\right. \\
& \left.\times \exp (-i \varepsilon t) \bar{p}_{-1}(t)-\Omega_{2}(t) \exp (i \varepsilon t) \bar{p}_{1}^{*}(t)\right\} .
\end{aligned}
$$

In deriving the left-hand side of Eq. (65), the relation $\Omega_{2}(t) \approx \Omega_{20} \exp \left(-i \omega_{2} t\right)$ valid for $\sigma_{2}>>1$ has been employed. Eventually the expression of $\tilde{p}_{\alpha E}^{(-3)}(\omega)$ in the frequency domain is obtained as

$$
\begin{aligned}
\tilde{p}_{\alpha E}^{(-3)}(\omega)= & 8 \pi^{2}\left[\left(\omega-\varepsilon_{+}+i \Gamma / 2\right)\left(\omega-\varepsilon_{-}+i \Gamma / 2\right)\right]^{-1} \\
& \times \int d \zeta d \eta \widetilde{\Omega}_{1}^{*}(\zeta) \widetilde{\Omega}_{2}(\eta) \widetilde{\Omega}_{2}(\zeta-\eta+\omega) \\
& \times\left(\frac{\mu_{\alpha}^{*}(E)}{\varepsilon-\eta-i \Gamma / 2}+\frac{\mu_{\alpha}(E)\left[S_{\alpha \alpha}^{(-)}(E)\right]^{*}}{\varepsilon-\zeta+i \Gamma / 2}\right),
\end{aligned}
$$

where $\varepsilon_{ \pm}=\left(\varepsilon+\omega_{2} \pm \Omega_{0}\right) / 2$ with $\Omega_{0}=\sqrt{\left(\varepsilon-\omega_{2}\right)^{2}+8 \Omega_{20}^{2}}$. Equation (67) depends linearly on $\Omega_{10}$, while effects of $\Omega_{20}$ are treated nonperturbatively. $\tilde{p}_{\alpha E}^{(-3)}$ obviously has two poles at $\omega=\varepsilon_{ \pm}-i \Gamma / 2$, which is reminiscent of the AT doublet ${ }^{33,35}$ because of $\left|\varepsilon_{+}-\varepsilon_{-}\right|=\Omega_{0}$ if $\Omega_{0}$ is considered the Rabi frequency.

As is provided in Appendix B, applying to the integrals of Eq. (67) the steepest-descent method ${ }^{59}$ that is ensured as far as $\sigma_{2} \gg 1$, a closed analytic expression of $\tilde{p}_{\alpha E}^{(-3)}$ is derived. Here the Fano model for the case of one-open and one-closed channels ${ }^{1}$ is employed and $\mu_{\alpha}(E)$ and $S_{\alpha \alpha}^{(-)}(E)$ are expressed by $^{60}$

$$
\mu_{\alpha}(E)=\mu_{b g} \exp (i \theta) \frac{\epsilon+q}{\epsilon-i}
$$

and $^{61}$

$$
S_{\alpha \alpha}^{(-)}(E)=\frac{\epsilon+i}{\epsilon-i}
$$


where $q$ is a Fano parameter, $\mu_{b g}$ means a dipole moment due to a background continuum being almost energy independent, $\epsilon=\left(\varepsilon-\varepsilon_{r}\right) /(\gamma / 2)$ with $\varepsilon_{r}$ a FR energy position and $\gamma$ a full width at half maximum(FWHM) of the FR, and $\theta$ $=-\arctan (1 / q)+\theta_{0}$ with $\theta_{0}$ an overall unknown phase constant. ${ }^{60}$ Linear absorption spectra of FR given by the Fano model is depicted in Fig. 3(a) assuming $\theta_{0}=0$. For $q$ $=0$ and -1000 , the profiles are symmetric. The former case $(q=0)$ corresponds to the extreme FR limit having an obvious transparent window. On the other hand, the latter $(q=$ -1000) corresponds to the weak FR limit with a Lorentzian shape, however, no discernible window. The case of $|q| \gg 1$ is thus considered a pure bound state rather than the FR, aside from a finite spectral width. ${ }^{62}$ Profiles of other $q$ values are asymmetric.

In Fig. 3(b), the SRFWM signals, $|\widetilde{P}(\omega, \tau)|^{2}$, thus obtained are shown for some $q$ values in the resonant excitations that $\omega_{1}=\omega_{2}=\varepsilon_{r}$, where $\theta_{0}=0$ is assumed for simplicity (see Appendix C). The signals exhibit the AT-like doublet and the relative intensities of both lobes depend strongly upon $q$, resulting in asymmetric spectral profiles except for $q=0$ and -1000 , in which the signals are symmetric. A sign change of $q$ results just in exact reversal of each lobe with respect to $\varepsilon=\varepsilon_{r}$. It is found that $q$ values rendering the linear absorption spectra symmetric (asymmetric) also induce symmetric (asymmetric) shapes of SRFWM signals. This statement is intuitively understood following discussion given in Appendix C. Specifically, for $q=-1000$ corresponding to a bound-state transition, these symmetric acStark sidebands are common to a two-level atomic system. ${ }^{33}$ As has been seen in Ref. 60, a $q$ value indicates an interference between contributions of an open channel and a closed channel, and hence the asymmetric doublet manifested here arises from phase modulation due to this Fano coupling through the $q$ value. In passing, a $q$ value that is obtained by fitting the Fano formula of Eq. (68) to the main peak of $0(1 s)$ in the calculated spectra of Fig. 1(b) is almost -5 , which has been taken into account in Fig. 3(b).

The associated TRFWM signals, $|P(t, \tau)|^{2}$, for several $q$ values are depicted in Fig. 3(c), where $\theta_{0}=0$ is assumed again. These spectra show the Rabi oscillations that are temporally damped due to dephasing with a common period, however, with different amplitudes. The dissimilarity observed in SRFWM is also reflected in the TRFWM signal having such a different damping pattern. The signal for $q$ $=0$ vanishes faster than that for $q=-1000$, while the corresponding SRFWM profiles are very much alike to each other. Specifically, the former signal is sustained up to no more than $t \approx 1.3 \mathrm{ps}$, and the latter signal is still discerned around $t \approx 2.2 \mathrm{ps}$. For $q=-5$ and -3 , the amplitudes are more enhanced than these two cases. In TRFWM, therefore, the Rabi system relevant to a FR state (with a finite $q$ ) is distinguished from that for a bound state (with $|q| \gg 1$ ).

\section{RESULTS AND DISCUSSION}

Now let us move to the SRFWM signals provided by the fully numerical calculations for the sample of $34 \AA-G a A s / 17$ $\AA-\mathrm{Al}_{0.3} \mathrm{Ga}_{0.7} \mathrm{As}$ superlattices with a bias field of $25 \mathrm{kV} / \mathrm{cm}$
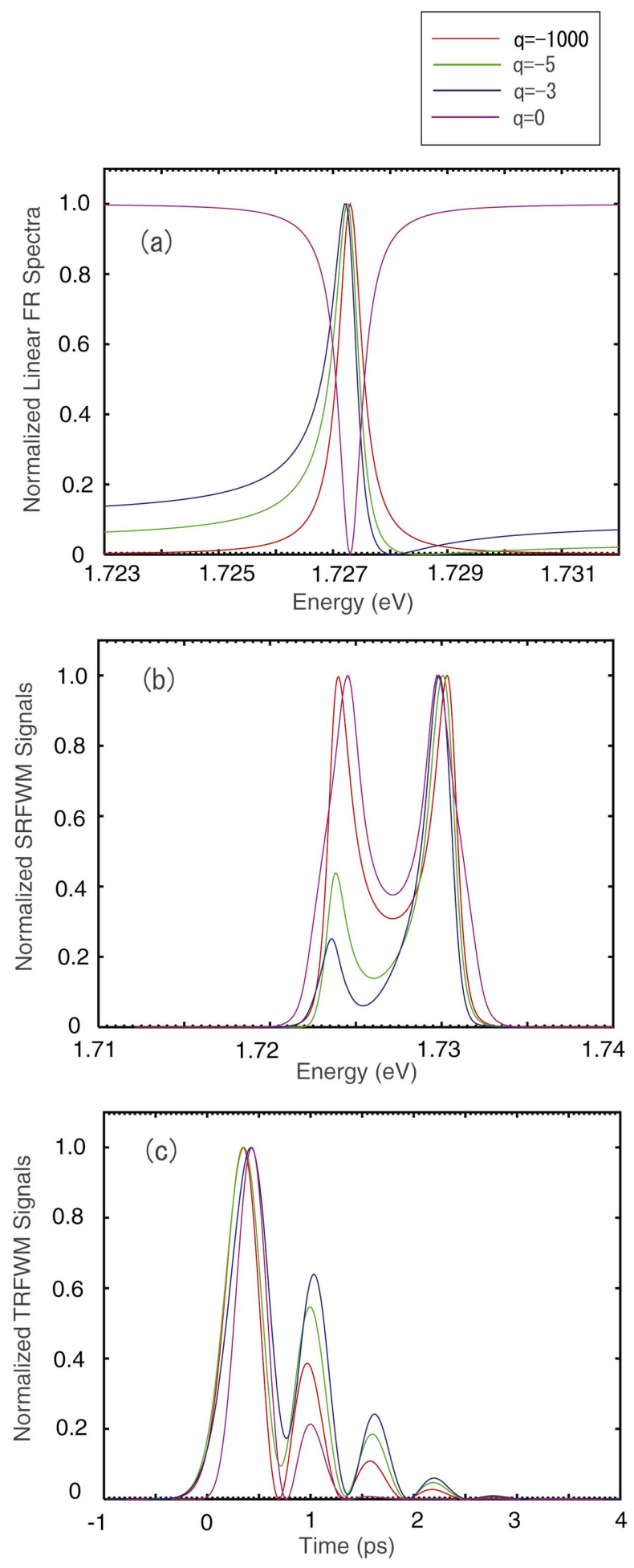

FIG. 3. (Color) (a) Normalized linear spectra of Eq. (17) obtained by the Fano model vs incident photon energies for $q=$ $-1000,-5,-3$ and 0 . (b) Normalized SRFWM spectra, $|\widetilde{P}(\omega, \tau)|^{2}$, obtained by the model calculations vs the energy $\omega$ for these $q$ values in resonant excitations of $\omega_{1}=\omega_{2}=\varepsilon_{r}=1.7273 \mathrm{eV}$. Other setup parameters are $F_{10}=1 \mathrm{kV} / \mathrm{cm}, F_{20}=100 \mathrm{kV} / \mathrm{cm}, \sigma_{1}$ $=\sigma_{2}=0.5 \mathrm{ps}, \quad T_{1}=T_{2}=1 \mathrm{ps}, \quad \tau=0.8 \mathrm{ps}$, and the FR width (FWHM) $0.5 \mathrm{meV}$. (c) Normalized TRFWM spectra, $|P(t, \tau)|^{2}$, corresponding to Fig. 3 (b) vs elapse timed $t$. Curves for different $q$ 's are distinguished by colors in all panels. 


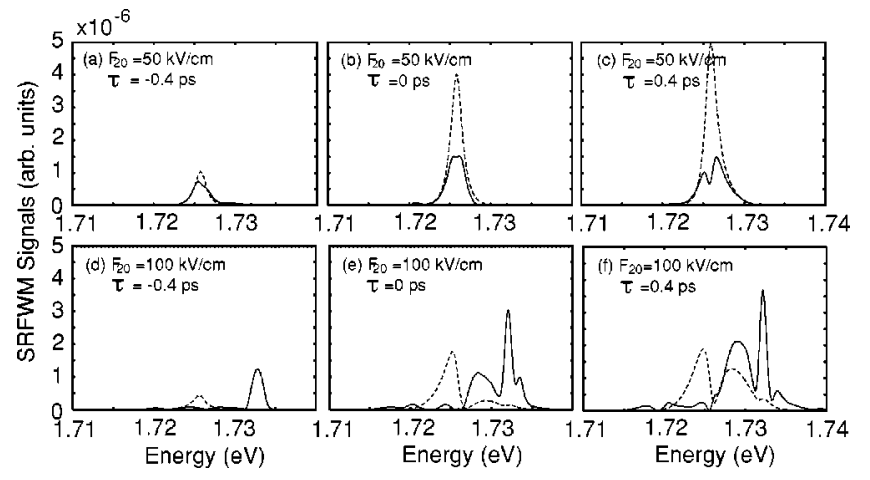

FIG. 4. SRFWM spectra, $|\widetilde{P}(\omega, \tau)|^{2}$, obtained by the fully numerical calculations vs the energy $\omega$ for different $\tau$ 's in resonant excitations of $\omega_{1}=\omega_{2}=\varepsilon_{r}=1.7273 \mathrm{eV}$ with (a) $-(\mathrm{c}) \quad F_{20}$ $=50 \mathrm{kV} / \mathrm{cm}$ and (d) - (f) $F_{20}=100 \mathrm{kV} / \mathrm{cm}$. Other setup parameters are $F_{10}=1 \mathrm{kV} / \mathrm{cm}, \sigma_{1}=\sigma_{2}=0.5 \mathrm{ps}$, and $T_{1}=T_{2}=1 \mathrm{ps}$. Solid (dashed) curves stand for the spectra with (without) the many-body effect, where (a) and (d) are for $\tau=-0.4$ ps (the pulse 2 precedes the pulse 1), (b) and (e) for $\tau=0 \mathrm{fs}$, and (c) and (f) for $\tau=0.4 \mathrm{ps}$ (the pulse 1 precedes the pulse 2), respectively.

along the crystal-growth direction, which is the same as the sample for the linear spectra of Fig. 1(b). The number of partial waves included is set $l_{\text {max }}=10$, and the whole effects of the HF Coulomb exchange are incorporated without assuming any additional imposition upon $\sigma_{j}$ and $F_{j 0}$. The dominant-channel approximation is still adopted, which is valid in the present sample. Furthermore, evaluations of $\mu_{\alpha E}, \varphi_{\lambda \lambda^{\prime} \alpha}^{E}$, and $S_{\alpha \beta}^{(-)}(E)$, required in Eqs. (41) and (42), resort to the linear-response theory for the excitonic FR described in Sec. II A, not to the simple Fano model employed in Sec. III. A frequency distribution of the pulse 2, with $\sigma_{2}$ $=500 \mathrm{fs}$ and tuned resonantly to $\varepsilon_{r}=1.7273 \mathrm{eV}$ is also depicted in Fig. 1(b). The pump field coherently excites most parts of the FR state of $0(1 s)$, and slightly overlaps with adjacent FR peaks such as $-1(3 s)$ and $0(2 s)$.

The calculated SRFWM signals are shown in Fig. 4, where the solid and dashed curves represent traces with and without the many-body effects, respectively, for three delayed times of $\tau=-0.4,0$, and $0.4 \mathrm{ps}$, with $T_{2}=1 \mathrm{ps}$. Figures $4(\mathrm{a}-\mathrm{c})$ show the results for $F_{10}=1 \mathrm{kV} / \mathrm{cm}$ and $F_{20}$ $=50 \mathrm{kV} / \mathrm{cm}$. Hereafter, the signals denoted by the solid and dashed lines of Fig. 4(c) are cited as the signals, $S_{c}$ and $S_{c}^{\prime}$, respectively. The profiles without the many-body effect are almost Lorentzian, whereas those with this effect are modulated to some extent and slight doublet structures are discerned, especially, for $\tau=0.4 \mathrm{ps}$. Incidentally, for a still smaller pumping field, for instance, $F_{20}=1 \mathrm{kV} / \mathrm{cm}$, spectra are little affected by the many-body Coulomb exchange and the resulting profiles remain Lorentzian for polarization dephasing of $T_{2}=1$ ps concerned here. For $T_{2}$ greater than 1 ps, comparable to or smaller than the FR width of nearly 0.5 meV of the peak $0(1 s)$ in Fig. 1(b), it would be likely that a SRFWM signal reflects a generic pattern of the Fano profile of the linear spectra. Otherwise a detailed asymmetry pattern seems to be smeared out by more rapid dephasing.

By comparing with Figs. 4(a-c), it is recognized that the many-body effect is augmented with increase of pumping intensity in Figs. 4(d-f), resulting in marked modification of the spectra. Hereafter the signals denoted by the solid and dashed lines of Fig. 4(f) are cited as the signals, $S_{f}$ and $S_{f}^{\prime}$, respectively. The overall shape of the signal of $S_{f}^{\prime}$ is akin to that of the signal of $q=-5$ in Fig. 3(b), aside from a slight variance of a relative intensity of the two sidebands, due presumably to the fact that the model calculations described in Sec. III are ensured for $\sigma_{2} \gg 1$. The main profile of the curve for the signal $S_{f}$ is shifted toward the high-energy side. Furthermore, the relative intensity of the two lobes is reversed from the trace of $S_{f}^{\prime}$, that is, the right lobe is stronger than the left one in $S_{f}$, and vice versa in $S_{f}^{\prime}$. Such asymmetry reversal also accompanies prominent changes of spectral widths: the right lobe is still narrower than the left one, whereas the widths of the two sidebands in $S_{f}^{\prime}$ are almost equal.

The three characteristics of the dressed FR states, namely, asymmetry reversal, spectral narrowing, and blue shift, are found here. The first two are unequivocally attributed to interplays between phase modulation induced by the FR couplings and the many-body effects. The phase modulation also contributes to forming the asymmetry in the AT-like doublet through the $q$ value, as was discussed in the preceding section. Phase-shift information is included in the FR wave functions required to evaluate the Coulomb exchange matrices, through $S_{n \alpha}^{(-)}$and $A_{n \alpha}^{(-)}$of Eqs. (20) and (21). ${ }^{63}$ Therefore the asymmetric AT-like doublet with the asymmetry reversal and the spectral narrowing is understood to be governed by a way of interference ascribable to the $q$ value and the $S$ matrix, and the many-body effect. As for the blue shift, the similar effect was already observed in the nonresonant acStark effect in the literature given in Ref. 34, and hence this would be due mostly to temporal evolution of the repulsive exciton-exciton interactions [see Eq. (43)].

The asymmetric doublet is also confirmed in the resonant ac-Stark splitting of an exciton bound state induced by coherent excitation of the absorption edge of a lowest-lying continuum state together with the bound state. ${ }^{29}$ Nevertheless, its origin is different from the present one. In the former, the continuum is structureless, and it is expressed as a real sinusoidal wave without any interference effect with the bound state. Moreover, the right sideband is always dominant, and the asymmetry reversal never occurs to the best of our knowledge. On the other hand, in the latter, the FR continuum is sharply energy and phase-shift dependent, and the degree of asymmetry varies in complicated manners according to setup parameters employed in addition to the many-body effects. As for the spectral narrowing, it has not been pointed out yet in any preceding study of excitonic ac-Stark effects. Thus this would be qualified as a novel effect found in the dressed FR system.

Figure 5 shows SRFWM signals with different dephasing and pumping intensity from those of Fig. 4. As is indicated in Figs. 5(a-c), the signal no longer exhibits the AT-like doublet even for $F_{20}=100 \mathrm{kV} / \mathrm{cm}$, due to still stronger dephasing $\left(T_{2}=0.2 \mathrm{ps}\right)$ of polarizations. Thus, for the splitting to manifest itself, it is necessary that an effective Rabi energy, namely, an external pump field plus an internal renormalized 


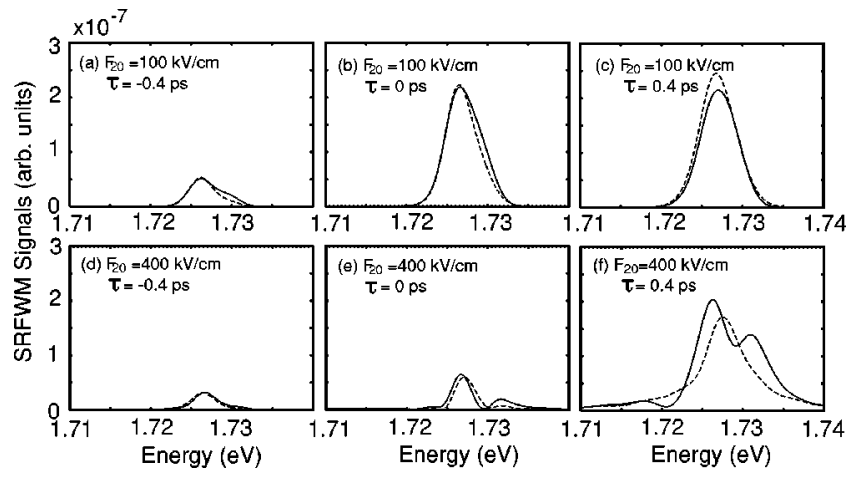

FIG. 5. The same as Fig. 4 but $T_{1}=T_{2}=0.2$ ps.

field, is greater than an inverse of dephasing time. Increasing the pumping strength to $F_{20}=400 \mathrm{kV} / \mathrm{cm}$, the doublet structure is retrieved in Figs. 5(d-f) if taking into account the many-body effect. However, in contrast with Figs. 4(d-f), it is found that the left lobe becomes dominant to the right one and the spectral narrowing is not observed. The strong dephasing suppresses appearance of the spectral narrowing, and spectral widths of the two lobes seem to be dominated by the dephasing time rather than the interplay between the phase modulation and the many-body effect, eventually being almost identical to each other.

Finally, one mentions to TRFWM signals depicted in Fig. 6. In Fig. 6(a) a solid (dashed) curve corresponds to the traces of $S_{c}\left(S_{c}^{\prime}\right)$ of Fig. 4(c). Similarly, in Fig. 6(b) a solid (dashed) curve corresponds to the traces of $S_{f}\left(S_{f}^{\prime}\right)$ of Fig. 4(f). It is understood that the same notations as $S_{c / f}$ and $S_{c / f}^{\prime}$ are also used for the TRFWM signals. For $F_{20}=50$ and 100 $\mathrm{kV} / \mathrm{cm}$, pulse areas defined as ${ }^{64}\left(\mu_{0} / 2\right) \int_{-\infty}^{\infty} F_{2}(t) d t$ are $\approx \pi / 2$ and $\pi$, respectively, thus the Rabi flopping is not complete. In fact it does not appear in $S_{c}^{\prime}$. However, the Rabi oscillation seems to manifest itself in $S_{f}^{\prime}$ during coherence time of an order of $T_{2}$, which is compatible with the existence of the AT-like doublet in $S_{f}^{\prime}$ of SRFWM. Moreover, there exist obvious quantum beat structures in $S_{c}^{\prime}$ and $S_{f}^{\prime}$ after about $2 \mathrm{ps}$, though it is considerably weakened in $S_{c}^{\prime}$. The beating period remains unaltered, being about $0.68 \mathrm{ps}$, in both cases despite the pumping strengths being different. This statement is contrasted with the Rabi oscillation depending linearly on the field strength. This period corresponds to an energy separation of $\Delta E$ of about $6 \mathrm{meV}$, which just coincides with the difference of the FR state of $0(1 s)$ from that of $0(2 s)$ that is coherently excited by a highenergy tail of the pumping laser [see Fig. 1(b)]. In addition, since the energy of $0(1 s)$ is separated from $-1(2 s)$ and $-1(3 s)$ by about $5-6 \mathrm{meV}$, these states are also likely to contribute to the quantum beat.

The Rabi flopping is also seen during the coherence time in $S_{c}$ and $S_{f}$ in addition to the quantum beat arising from $\Delta E$, which is somewhat modulated in an irregular manner due presumably to the many-body effect. Note that the period of the Rabi oscillation of $S_{f}$ looks almost half longer than that of $S_{f}^{\prime}$. This results from enhancement of the internal renormalized fields and thus the net Rabi energies during temporal evolution of the pulse 2 . This tendency of the time-

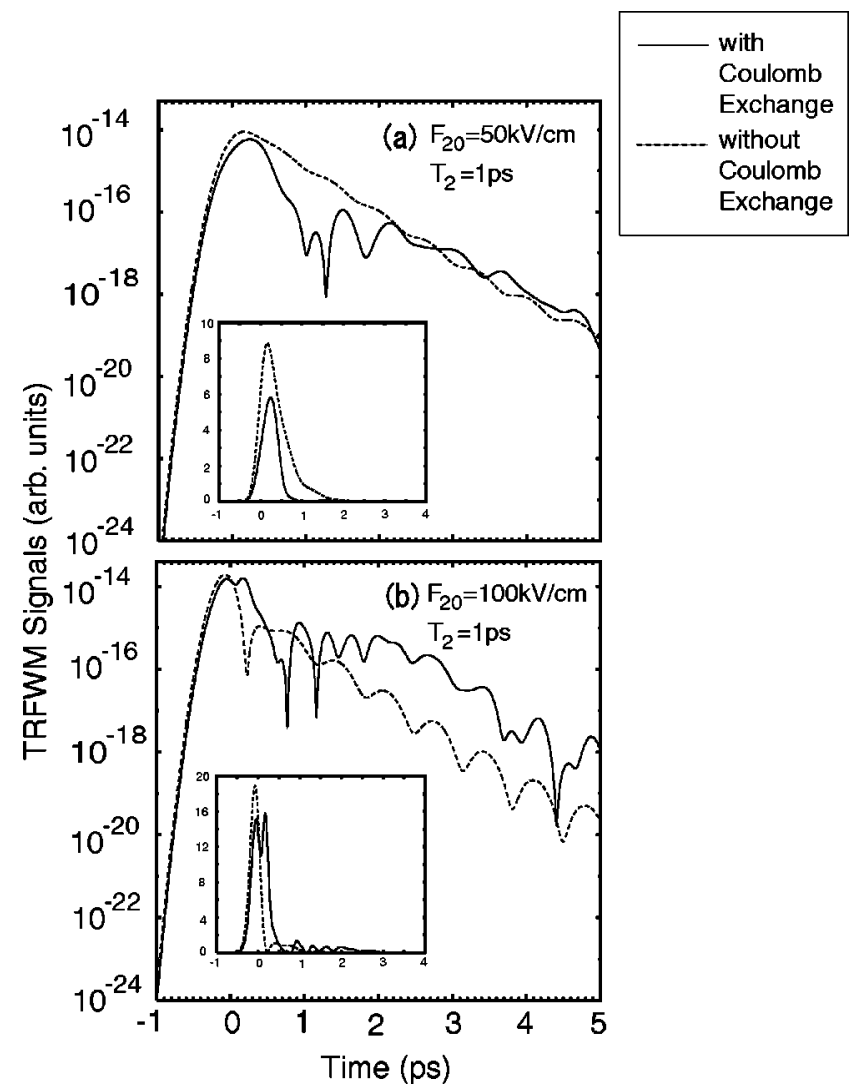

FIG. 6. TRFWM spectra, $|P(t, \tau)|^{2}$, obtained by the fully numerical calculations vs elapsed time $t$ for $\tau=0.4$ ps. Solid (dashed) curves represent the results with (without) the many-body effect, having the same employed setup parameters as those of Fig. 4. Insets are the same of the corresponding main panels but depicted in a linear scale for comparison.

evolution spectra resembles the Rabi-oscillation pattern already known in bound-state Rabi systems, ${ }^{18,26,27}$ and therefore only this behavior does not seem to uniquely feature the present dressed FR system. Recalling the model calculations and discussion regarding Fig. 3(c), it would be probable that the noted enhancement effect of an oscillation amplitude due to the Fano coupling contributes more or less to the spectral patterns of the present TRFWM signals as well.

\section{CONCLUDING REMARKS}

To summarize, the transient FWM spectra induced by resonant excitations of FR excitons are calculated by solving both analytically and numerically the SBE within the HF approximation. The asymmetric AT-like doublet emerges in the SRFWM signals. The degree of this asymmetry depends strongly upon a Fano- $q$ parameter and the many-body Coulomb exchange. Furthermore, the many-body effects also cause the asymmetry reversal, the spectral narrowing and the blue shift, all of which feature the present laser-driven FR exciton system. The findings would propose novel aspects for discriminating the dressed-semiconductor system from the dressed-atomic system. The pattern of the spectra is partially smeared out by faster dephasing, though the asymmetric AT-like doublet is retrieved by making the pumping field 
stronger. The Rabi oscillation relevant to this splitting also appears in the TRFWM signals. Following the results of the model calculations, it is likely that an amplitude of the oscillation is modified prominently with changing the $q$ value.

Finally three remarks will be made. The first is that in the present paper the Coulomb correlation beyond the mean-field approximation has not been taken into account, as was mentioned in Sec. I. However, it is likely that this effect modifies the results obtained herein to some extent. To the best of our knowledge, the Coulomb correlation has not been examined in the laser-driven FR system thus far irrespective of whether the laser is resonant or nonresonant and strong or weak. This is an open problem. The second is on the FWM study of FR reported by Siegner et al. ${ }^{6,7}$ They found that the decay of the TRFWM is related to dephasing. On the other hand, the decay of time-integrated FWM is much faster and almost independent of excitation densities. It was speculated that this phenomena arises from some sorts of destructive interference due to FR in conjunction with nonlinearity and the manybody Coulomb effect. This still remains raveled. The third comment is on possibility of photon echo in the laser-driven FR system. ${ }^{65}$ It is usually understood that without inhomogeneous broadening the photon echo is not generated, unless excitation is so strong that a bound-state level overlaps with the edge of continuum onset due to blue shift. ${ }^{66}$ In FR the discrete level is always degenerate to lower-lying continua playing a role of inhomogeneousity. Therefore, it is likely that the photon echo is discernible even in weak excitation. Indeed in view of Eq. (57) the FR distribution of $\mu_{\alpha}(E)$ fulfills the same role as the inhomogeneous broadening. The echo spectra are suppressed by dephasing, and hence manifestation of the echo in TRFWM depends on how fast dephasing is.

\section{ACKNOWLEDGMENT}

This research was partially supported by a Grant-in-Aid for Scientific Research (B) from Japan Society for the Promotion of Science.

\section{APPENDIX A: ON THE HF COULOMB EXCHANGE INTEGRALS}

In this appendix, the HF Coulomb exchange matrix element of $\mathcal{V}_{\alpha E ; \beta E^{\prime} ; \gamma E^{\prime \prime}}$ of Eq. (47) is reduced to a form feasible in a practical computation. The obtained results can be straightforward applied to the similar integrals of $\mathcal{V}_{\alpha E ; \beta E^{\prime} ; \gamma E^{\prime \prime}}^{\prime}$ and $\mathcal{V}_{\alpha E ; \beta E^{\prime} ; \gamma E^{\prime \prime}}^{\prime \prime}$ This task is equivalent to evaluating the following expression:

$$
I=\int d \rho d \mathbf{x} f_{1}(\rho) V(\mathbf{x}) f_{2}(\mathbf{x}) f_{3}(\rho-\mathbf{x}),
$$

where the form of $V(\mathbf{x})$ given right below Eq. (54) has been replaced by a screened Coulomb potential, $\exp (-\kappa|\mathbf{x}|) /(\epsilon|\mathbf{x}|)$, by introducing a small and positive parameter $\kappa$ for numerical stabilization. A numerical result is insensitive to a choice of $\kappa$ as far as it is small enough not to affect the result. A function of $f_{j}(\rho)(j=1-3)$ relevant to the radial wave function of $\hat{F}_{n_{j} \alpha_{j}}^{E_{j}}(|\rho|) / \sqrt{|\rho|}$ is of the form

$$
f_{j}(\rho)=\frac{1}{\sqrt{2 \pi}}|\rho|^{\left(N_{j}-1\right) / 2} \exp \left(i k_{j}|\rho|\right)
$$

in light of Eqs. (20) and (21). Here $1 / \sqrt{2 \pi}$ is ascribable to Eq. (9) for the $s$-radial symmetry $(m=0)$, and $\left|k_{j}\right|$ $=\sqrt{2 m_{\|}\left|E_{j}-U_{n_{j}}(\infty)\right|} ; k_{j}$ is a real number for $n_{j}$ open and a pure imaginary number with $\operatorname{Im}\left(k_{j}\right)>0$ for $n_{j}$ closed. In addition, $N_{j}=0$ for $n_{j}$ open and $N_{j}=1$ for $n_{j}$ closed.

By use of a set of confocal elliptic coordinates $\{\mu, \nu\}$ defined by ${ }^{67}$

$$
\mu=\frac{|\rho|+|\rho-\mathbf{x}|}{|\mathbf{x}|}, \quad \nu=\frac{|\rho|-|\rho-\mathbf{x}|}{|\mathbf{x}|},
$$

we have

$$
d \rho=\left(\frac{|\mathbf{x}|}{2}\right)^{2} \frac{\mu^{2}-\nu^{2}}{\left[\left(\mu^{2}-1\right)\left(1-\nu^{2}\right)\right]^{1 / 2}} d \mu d \nu .
$$

Putting Eqs. (A2)-(A4) into Eq. (A1) yields

$$
\begin{aligned}
I= & \frac{1}{\sqrt{2 \pi} \epsilon}\left(\frac{1}{2}\right)^{\left(N_{1}+N_{3}\right) / 2} \int_{1}^{\infty} d \mu \int_{-1}^{1} \frac{d \nu}{\sqrt{1-\nu^{2}}} \\
& \times\left\{\frac{(\mu+\nu)^{\left(N_{1}+1\right) / 2}(\mu-\nu)^{\left(N_{3}+1\right) / 2}}{\sqrt{\mu^{2}-1}}\right\} \\
& \times \int_{0}^{\infty} d|\mathbf{x}||\mathbf{x}|^{\left(N_{1}+N_{2}+N_{3}+1\right) / 2} \\
& \times \exp \left[\left(i k_{2}-\kappa+i \frac{k_{1}+k_{3}}{2} \mu+i \frac{k_{1}-k_{3}}{2} \nu\right)|\mathbf{x}|\right] .
\end{aligned}
$$

It is noted that the integral over $\mu$ is divergent when $N_{1}$ $=N_{3}=0$ and $k_{1}+k_{3}=0$, since the part of the integrand parenthesized by curly brackets becomes unity for $\mu \rightarrow \infty$. Such a singularity can be removed by subtracting from this integral over $\mu$ and add to it

$$
\int_{1}^{\infty} d \mu \exp \left(i \frac{k_{1}+k_{3}}{2}|\mathbf{x}| \mu\right)=\frac{2 \pi}{|\mathbf{x}|} \delta\left(k_{1}+k_{3}\right) .
$$

Moreover, the integral over $|\mathbf{x}|$ is readily implemented to be of the form of the $\Gamma$ function. Eventually Eq. (A1) becomes

$$
\begin{aligned}
I= & \frac{1}{\sqrt{2 \pi} \epsilon}\left(\frac{1}{2}\right)^{\left(N_{1}+N_{3}\right) / 2} \int_{1}^{\infty} d \mu \int_{-1}^{1} \frac{d \nu}{\sqrt{1-\nu^{2}}} \\
& \times\left\{\frac{(\mu+\nu)^{\left(N_{1}+1\right) / 2}(\mu-\nu)^{\left(N_{3}+1\right) / 2}-\delta_{N_{1} 0} \delta_{N_{3} 0} \delta\left(k_{1}+k_{3}\right)}{\sqrt{\mu^{2}-1}}\right\} \\
& \times\left(-i k_{2}+\kappa-i \frac{k_{1}+k_{3}}{2} \mu\right.
\end{aligned}
$$




$$
\begin{aligned}
& \left.-i \frac{k_{1}-k_{3}}{2} \nu\right)^{-\left(N_{1}+N_{2}+N_{3}+3\right) / 2} \Gamma\left(\frac{N_{1}+N_{2}+N_{3}+3}{2}\right) \\
& +2 \pi \delta_{N_{1} 0} \delta_{N_{3} 0} \delta\left(k_{1}+k_{3}\right) \Gamma\left(\frac{N_{2}+1}{2}\right) \int_{-1}^{1} \frac{d \nu}{\sqrt{1-\nu^{2}}} \\
& \times\left(-i k_{2}+\kappa-i k_{1} \nu\right)^{-\left(N_{2}+1\right) / 2}
\end{aligned}
$$

Making replacements that $\mu=\cosh t$ and $\nu=\cos \theta$, the integrations over $t$ and $\theta$ become numerically tractable, and feasible resorting to a standard quadrature formula.

\section{APPENDIX B: ON THE INTEGRAL OF EQ. (67)}

Equation (67) is reduced by use of the steepest-descent method. ${ }^{59}$ Since the pulses are Gaussian, it suffices to deal with the following integral:

$$
J=\int d \eta A_{2}\left(\eta-\omega_{2}\right) \frac{1}{\varepsilon-\eta-i \Gamma / 2} K(\eta),
$$

where

$$
K(\eta)=\int d \zeta A_{1}^{*}\left(\zeta-\omega_{1}\right) A_{2}\left(\zeta-\eta+\omega-\omega_{2}\right),
$$

with $t_{1}=-\tau, t_{2}=0$, and

$$
A_{j}(\eta)=\exp \left[i\left(\omega_{j}-\eta\right) t_{j}-\left(\frac{\sigma_{j}\left(\omega_{j}-\eta\right)}{2}\right)^{2}\right] .
$$

First $K$ is readily obtained by

$$
K(\eta)=c \exp [-a(\eta)]
$$

where

$$
c=\frac{2 \sqrt{\pi}}{\sqrt{\sigma_{1}^{2}+\sigma_{2}^{2}}}
$$

and

$$
\begin{aligned}
a(\eta)= & -\frac{1}{4\left(\sigma_{1}^{2}+\sigma_{2}^{2}\right)}\left[\sigma_{1}^{2} \omega_{1}+\sigma_{2}^{2}\left(\omega_{2}+\eta-\omega\right)+2 i\left(t_{1}-t_{2}\right)\right]^{2} \\
& +\frac{1}{4} \sigma_{1}^{2} \omega_{1}^{2}+\frac{1}{4} \sigma_{2}^{2}\left(\omega_{2}+\eta-\omega\right)^{2} \\
& +i\left[\omega_{1} t_{1}-\left(\omega_{2}+\eta-\omega\right) t_{2}\right] .
\end{aligned}
$$

Putting Eq. (B4) into Eq. (B1) provides the form

$$
J=c \int_{-\infty}^{\infty} d \eta \exp \left[-\sigma_{2} \mathcal{F}(\eta)\right]
$$

where

$$
\begin{aligned}
\sigma_{2} \mathcal{F}(\eta)= & -i\left(\omega_{2}-\eta\right) t_{2}+\left(\frac{\sigma_{2}\left(\omega_{2}-\eta\right)}{2}\right)^{2} \\
& +\ln (\varepsilon-\eta-i \Gamma / 2)+a(\eta)
\end{aligned}
$$

Since $\operatorname{Re}[\mathcal{F}(\eta)] \rightarrow \infty$ with $\sigma_{2} \rightarrow \infty$ is satisfied, Eq. (B7) is evaluated by employing the steepest-descent method. Hence $\mathcal{F}$ is approximated by

$$
\mathcal{F}(\eta) \approx \mathcal{F}\left(\eta_{k}\right)+\frac{1}{2} \mathcal{F}^{\prime \prime}\left(\eta_{k}\right)\left(\eta-\eta_{k}\right)^{2},
$$

where there are two saddle points in the complex $\eta$ plane, denoted as $\eta_{k}(k=1,2)$, and

$$
\mathcal{F}^{\prime \prime}\left(\eta_{k}\right)=\left[\frac{d^{2} \mathcal{F}(\eta)}{d \eta^{2}}\right]_{\eta=\eta_{k}}
$$

Finally we obtain

$$
J=c \sum_{k=1,2} \exp \left[-\sigma_{2} \mathcal{F}\left(\eta_{k}\right)\right]\left(\frac{2 \pi}{\sigma_{2} \mathcal{F}^{\prime \prime}\left(\eta_{k}\right)}\right)^{1 / 2} .
$$

\section{APPENDIX C: SPECTRAL PROFILES IN SRFWM}

The origin of symmetry and asymmetry in SRFWM is intuitively understood in the following. First Eq. (67) is rewritten as

$$
\begin{aligned}
\tilde{p}_{\alpha E}^{(-3)}(\omega)= & 8 \pi^{2}\left[\mu_{\alpha}^{\prime}(E)\right]^{*}\left[\left(\omega-\varepsilon_{+}+i \Gamma / 2\right)\right. \\
& \left.\times\left(\omega-\varepsilon_{-}+i \Gamma / 2\right)\right]^{-1} F(\omega),
\end{aligned}
$$

where

$$
\begin{aligned}
F(\omega)= & \int d \zeta d \eta \widetilde{\Omega}_{1}^{*}(\zeta) \widetilde{\Omega}_{2}(\eta) \widetilde{\Omega}_{2}(\zeta-\eta+\omega) \\
& \times\left[\frac{\exp (-i \theta)}{\varepsilon-\eta-i \Gamma / 2}+\frac{\exp (i \theta)}{\varepsilon-\zeta+i \Gamma / 2}\right]
\end{aligned}
$$

and $\theta$ and $\mu_{\alpha}^{\prime}(E)$ have been defined that $\theta=-\arctan (1 / q)$ $+\theta_{0}$ with $\theta_{0}$ a constant ${ }^{60}$ and $\mu_{\alpha}^{\prime}(E)=(\epsilon+q) /(\epsilon-i)$, respectively. It is $F(\omega)$ that dominates the asymmetry in spectra, as is seen right below, and the prefactor of Eq. (C1) merely provides a symmetric profile. Suppose that $\widetilde{\Omega}_{2}(\eta)$ $=\widetilde{\Omega}_{20} \delta\left(\eta-\omega_{2}\right)$ and that a peaking approximation of $\varepsilon$ $\approx \omega_{2}$ is made partially in $F(\omega)$. The former is accurate for $\sigma_{2} \rightarrow \infty$, and the latter is due to that the natural width of FR $\gamma$ (usually of the order of $0.1 \mathrm{meV}$ ) is much smaller than the separation $\Omega_{0}$ between the AT-like sidebands. Hence $F(\omega)$ is recast into

$$
F(\omega) \approx \widetilde{\Omega}_{1}^{*}\left(2 \omega_{2}-\omega\right) \widetilde{\Omega}_{20}^{2} \frac{\left(\omega-\omega_{2}\right) \exp (-i \theta)+\Gamma \sin \theta}{\left(\varepsilon-\omega_{2}-i \Gamma / 2\right)\left(\omega-\omega_{2}+i \Gamma / 2\right)} .
$$

If a relation that $|F(\omega)|^{2}=\left|F\left(2 \omega_{2}-\omega\right)\right|^{2}$ is satisfied, the overall profile is symmetric with respect to the FR position $\varepsilon_{r}=\omega_{2}$, otherwise it is asymmetric. The spectral pattern is determined solely by the numerator of Eq. (C3). Here the unknown constant of $\theta_{0}$ is considered zero for simplicity in the following discussion in this appendix. In practice, this phase factor is unambiguously determined by imposition of the scattering wave boundary condition of Eq. (6) on the FR wave function. For $|q|=\infty$ corresponding to a bound-state 
case and $\theta=0$, it is found that the symmetric spectra are obtained. The similar situation holds for the case of $q=0$, corresponding to $\theta=-\pi / 2$. For other $\theta$ 's, however, it is seen that $F(\omega)$ leads to an asymmetric profile. Therefore it is concluded that the asymmetry arises from interference due to the phase of $\theta$. Following the above discussion, it is found that the maximum asymmetry is realized for $\theta= \pm \pi / 4$ corresponding to $q=\mp 1$. In a more accurate evaluation made in Sec. III, the maximum asymmetry appears around $|q|$ $=2-3$ [see Fig. 3(b)].
${ }^{1}$ U. Fano, Phys. Rev. 124, 1866 (1961).

${ }^{2}$ M.V. Fedorov and A.E. Kazakov, Prog. Quantum Electron. 13, 1 (1989).

${ }^{3}$ Z. Deng and J.H. Eberly, Phys. Rev. A 36, 2750 (1987).

${ }^{4}$ P.L. Knight, M.A. Lauder, and B.J. Dalton, Phys. Rep. 190, 1 (1990). LIA differs from LICS in that the embedded state is not laser induced, but inherent in an original unperturbed atom.

${ }^{5}$ T. Meier, A. Schulze, P. Thomas, and H. Vaupel, Phys. Rev. B 51, 13977 (1995).

${ }^{6}$ U. Siegner, M.-A. Mycek, S. Glutsch, and D.S. Chemla, Phys. Rev. Lett. 74, 470 (1995).

${ }^{7}$ U. Siegner, M.-A. Mycek, S. Glutsch, and D.S. Chemla, Phys. Rev. B 51, 4953 (1995).

${ }^{8}$ C.P. Holfeld, F. Löser, M. Sudzius, K. Leo, D.M. Whittaker, and K. Köhler, Phys. Rev. Lett. 81, 874 (1998).

${ }^{9}$ T.W. Canzler, C.P. Holfeld, F. Löser, V.G. Lyssenko, K. Leo, D.M. Whittaker, and K. Köhler, Physica E 10, 593 (2001).

${ }^{10}$ D.Y. Oberli, G. Böhm, G. Weimann, and J.A. Brum, Phys. Rev. B 49, 5757 (1994).

${ }^{11}$ S. Bar-Ad, P. Knor, M.V. Marquenzini, S. Mukamel, and D.S. Chemla, Phys. Rev. Lett. 78, 1363 (1997).

${ }^{12}$ D.M. Whittaker, J. Phys. IV 5, 199 (1993).

${ }^{13}$ D.M. Whittaker, Europhys. Lett. 31, 55 (1995).

${ }^{14}$ K. Hino, Phys. Rev. B 62, R10626 (2000).

${ }^{15}$ K. Hino, Phys. Rev. B 64, 075318 (2001), and see also references cited therein.

${ }^{16}$ R. Winkler, Phys. Rev. B 51, 14395 (1995), and see also references cited therein.

${ }^{17}$ S. Glutsch, D.S. Chemla, and F. Bechstedt, Phys. Rev. B 54, 11 592 (1996); S. Glutsch and F. Bechstedt, ibid. 57, 11887 (1998).

${ }^{18}$ S.T. Cundiff, A. Knor, J. Feldmann, S.W. Koch, E.O. Göbel, and H. Nickel, Phys. Rev. Lett. 73, 1178 (1994).

${ }^{19}$ L. Schultheis, J. Kuhl, A. Honold, and C.W. Tu, Phys. Rev. Lett. 57, 1635 (1986).

${ }^{20}$ A. Honold, L. Schltheis, J. Kuhl, and C.W. Tu, Phys. Rev. B 40, 6442 (1989).

${ }^{21}$ M.U. Wehner, D. Steinbach, and M. Wegener, Phys. Rev. B 54, R5211 (1996).

${ }^{22}$ D. Birkedal, V.G. Lyssenko, J.M. Hvam, and K. El Sayed, Phys. Rev. B 54, 14250 (1996).

${ }^{23}$ J.M. Shacklette and S.T. Cundiff, Phys. Rev. B 66, 045309 (2002).

${ }^{24}$ M. Lindberg and R. Binder, Phys. Rev. Lett. 75, 1403 (1995).

${ }^{25}$ H. Giessen, A. Knor, S. Haan, S.W. Koch, S. Linden, J. Kuhl, M. Hetterich, M. Grün, and C. Klingshirn, Phys. Rev. Lett. 81, 4260 (1998).

${ }^{26}$ A. Schülzgen, R. Binder, M.E. Donovan, M. Lindberg, K. Wundke, and H.M. Gibbs, Phys. Rev. Lett. 82, 2346 (1999).

${ }^{27}$ R. Binder and M. Lindberg, Phys. Rev. B 61, 2830 (2000).

${ }^{28}$ C. Ciuti and F. Quochi, Solid State Commun. 107, 715 (1998).
${ }^{29}$ M. Saba, F. Quochi, C. Ciuti, D. Martin, J.-L. Staehli, B. Deveaud, A. Mura, and G. Bongiovanni, Phys. Rev. B 62, R16 322 (2000).

${ }^{30}$ F. Quochi, G. Bongiovanni, A. Mura, J.L. Staehli, M.A. Dupertuis, R.P. Stanley, U. Oesterle, and R. Houdré, Phys. Rev. Lett. 80, 4733 (1998).

${ }^{31}$ F. Quochi, C. Ciuti, G. Bongiovanni, A. Mura, M. Saba, U. Oesterle, M.A. Dupertuis, J.L. Staehli, and B. Deveaud, Phys. Rev. B 59, R15 594 (1999).

${ }^{32}$ F. Quochi, M. Saba, C. Ciuti, R.P. Stanley, R. Houdré, U. Oesterle, J.L. Staehli, B. Deveaud, G. Bongiovanni, and A. Mura, Phys. Rev. B 61, R5113 (2000).

${ }^{33}$ P.L. Knight and P.W. Milonni, Phys. Rep. 66, 21 (1980).

${ }^{34}$ This differs from the so-called excitonic optical Stark effect observed under a nonresonant virtual-excitation condition, where the Rabi optical nutation is considered unimportant and the phenomena are well understood within a perturbation scheme. See, A. Mysyrowics, D. Hulin, A. Antonetti, A. Migus, W.T. Masselink, and H. Morkoc, Phys. Rev. Lett. 56, 2748 (1986); C. Ell, J.F. Müller, K. El Sayed, and H. Haug, ibid. 62, 304 (1989); W.H. Knox, D.S. Chemla, D.A.B. Miller, J.B. Stark, and S. Schmitt-Rink, ibid. 62, 1189 (1989).

${ }^{35}$ S.H. Autler and C.H. Townes, Phys. Rev. 100, 703 (1955).

${ }^{36}$ E.T. Jaynes and F.W. Cummings, Proc. IEEE 51, 89 (1963).

${ }^{37}$ S.M. Sadeghi and J. Meyer, J. Phys.: Condens. Matter 9, 7685 (1997).

${ }^{38} \mathrm{Ph}$. Tamarat, B. Lounis, J. Bernard, M. Orrit, S. Kummer, R. Kettner, S. Mais, and Th. Basché, Phys. Rev. Lett. 75, 1514 (1995).

${ }^{39}$ H. Haug and S. W. Koch, Quantum Theory of the Optical and Electronic Properties of Semiconductors (World Scientific, Singapore, 1993), Chap. 12.

${ }^{40}$ V.M. Axt, G. Bartels, and A. Stahl, Phys. Rev. Lett. 76, 2543 (1996).

${ }^{41}$ M. Hawton and D. Nelson, Phys. Rev. B 57, 4000 (1998).

${ }^{42}$ P.H. Bolivar, F. Wolter, A. Müller, H.G. Roskos, H. Kurz, and K. Köhler, Phys. Rev. Lett. 78, 2232 (1997).

${ }^{43}$ J.M. Lachaine, M. Hawton, J.E. Sipe, and M.M. Dignam, Phys. Rev. B 62, R4829 (2000).

${ }^{44}$ M. Dignam and M. Hawton, Phys. Rev. B 67, 035329 (2003).

${ }^{45}$ C. Sieh, T. Meier, F. Jahke, A. Knorr, S.W. Koch, P. Brick, M. Hübner, C. Ell, J. Prineas, G. Khitrova, and H.M. Gibbs, Phys. Rev. Lett. 82, 3112 (1999).

${ }^{46}$ A.L. Smirl, M.J. Stevens, X. Chen, and O. Buccaufusca, Phys. Rev. B 60, 8267 (1999).

${ }^{47}$ C. Ciuti, C. Piermarrocchi, V. Savona, P.E. Selbmann, P. Schwendimann, and A. Quattropani, Phys. Rev. Lett. 84, 1752 (2000).

${ }^{48}$ M.M. Dignam and J.E. Sipe, Phys. Rev. B 43, 4097 (1991).

${ }^{49}$ C. de Boor, A Practical Guides to Splines (Springer, Berlin, 1978), Chap. 9. 
${ }^{50}$ N. Linder, Phys. Rev. B 55, 13664 (1996).

${ }^{51}$ Q. Niu, X.-G. Zhao, G.A. Georgakis, and M.G. Raizen, Phys. Rev. Lett. 76, 4504 (1996).

${ }^{52}$ S.R. Wilkinson, C.F. Bharucha, K.W. Madison, Q. Niu, and M.G. Raizen, Phys. Rev. Lett. 76, 4512 (1996).

${ }^{53}$ M.B. Dahan, E. Peike, J. Reichel, Y. Castin, and C. Salomon, Phys. Rev. Lett. 76, 4508 (1996).

${ }^{54}$ M.C. Fischer, K.W. Madison, Q. Niu, and M.G. Raizen, Phys. Rev. A 58, R2648 (1998).

${ }^{55}$ K.W. Madison, M.C. Fischer, and M.G. Raizen, Phys. Rev. A 60, R1767 (1999).

${ }^{56}$ V.M. Axt and S. Mukamel, Rev. Mod. Phys. 70, 145 (1998).

${ }^{57}$ S. Yokojima, T. Meier, V. Chernyak, and S. Mukamel, Phys. Rev. B 59, 12584 (1999).

${ }^{58}$ W.M. Zhang, T. Meier, V. Chernyak, and S. Mukamel, Phys. Rev. B 60, 2599 (1999).

${ }^{59}$ P.M. Morse and H. Feshbach, Methods of Theoretical Physics (McGraw Hill, New York, 1953), Chap. 4.

${ }^{60}$ Equation (68) did not be given in the original paper of Fano (Ref. $1)$. It has been derived for the present problem of one open and one closed channels in the following. Based on the paper, B.W. Shore, Rev. Mod. Phys. 39, 439 (1967), $\mu_{\alpha}(E)$ is expressed as

$$
\mu_{\alpha}(E)=\frac{A}{\varepsilon-\varepsilon_{r}-i \gamma / 2}+B,
$$

where Shore's parameters, $A$ and $B$, are given by $A$ $\equiv A^{\prime} \exp \left(i \theta_{0}\right)$ and $B \equiv B^{\prime} \exp \left(i \theta_{0}\right)$. Here $A^{\prime}$ and $B^{\prime}$ have been defined real and complex numbers, respectively, since just a relative phase of the two is effective for a transition probability of $\left|\mu_{\alpha}(E)\right|^{2}$. The overall phase factor of $\theta_{0}$ is an unknown constant. $A$ represents a transition via a closed channel and $B$ stands for that via an open channel. Equating the above equation followed by taking a modulus square to $\left|\mu_{b g}\right|^{2}(q+\epsilon)^{2} /\left(\epsilon^{2}+1\right)$ provides the additional phase factor of $\exp [i\{-\arctan (1 / q)$ $\left.\left.+\theta_{0}\right\}\right]$ in Eq. (68) as well as the relations of $q$ $=\operatorname{Re}\left(B^{\prime}\right) A^{\prime} /\left(\left|B^{\prime}\right|^{2} \gamma / 2\right)$ and $\mu_{b g}=\left|B^{\prime}\right|^{2}$. In Fano's paper (Ref. $1), q$ is defined by $q=(\Phi|T| i) /\left[\pi V_{E}^{*}\left(\psi_{E}|T| i\right)\right]$, where $(\Phi|T| i)$ and $\left(\psi_{E}|T| i\right)$ mean transition matrices from an initial state of $i$ to a (modified) bound state $\Phi$ and a continuum state $\psi_{E}$ with an energy of $E$, respectively, and $V_{E}$ is an interaction between the bound and the continuum states. Extension to a problem of a number of open and closed channels is discussed in the Shore's paper.

${ }^{61}$ Bearing in mind $S_{\alpha \alpha}^{(-)}=\exp (-2 i \Delta)$, a FR phase shift $\Delta$ is given by $\cot \Delta=-\varepsilon$, following Fano's paper (Ref. 1).

${ }^{62}$ For an actual bound state, $\mu_{\alpha}(E)$ should be replaced by $\mu_{b} \delta(E$ $\left.-E_{b}\right)$ and $S_{\alpha \alpha}^{(-)}(E)$ by unity, where $\mu_{b}$ and $E_{b}$ indicate a dipole moment and a discrete energy associated with the bound state.

${ }^{63}$ It is noticed that a structure of the $S$ matrix obtained by the numerical calculation described in Sec. II A is in fact more complicated than that of Eq. (69). The latter is valid just in the close proximity of $\varepsilon_{r}$ and the $q$ dependence is approximately neglected. The $q$ dependence of the $S$ matrix is clearly seen, for instance, in autoionization induced by electron-atom collisions. Both elastic- and inelastic-scattering cross sections exhibit Fanotype distributions of an emitted electron. These are determined just by the $S$ matrix, and overall knowledge of wave functions are not necessary, differing from the associated photoionization cross section. Usually the $S$ matrix is more essential in FR than the $q$ value.

${ }^{64}$ P. Meystre and M. Sargent, III, Elements of Quantum Optics, 3rd ed. (Springer, Berlin, 1998) Chap. 12.

${ }^{65}$ K. Goto, K. Hino, and N. Toshima (unpublished); K. Goto, Master's thesis, University of Tsukuba, 2003.

${ }^{66}$ M. Lindberg, R. Binder, and S.W. Koch, Phys. Rev. A 45, 1865 (1992).

${ }^{67}$ H. Eyring, J. Walter, and G.E. Kimball, Quantum Chemistry (Wiley, New York, 1944), Chap. 11. 